\title{
Weak Turbulence Theory of Collisionless Trapped Electron Driven Drift Instability in Tokamaks
}

\author{
T. S. Hahm and W. M. Tang \\ Princeton Plasma Physics Laboratory, Princeton University \\ Princeton, N.J.08543
}

\begin{abstract}
The toroidal collisionless trapped electron modes are analyzed in the weak turbulence regime treating both ions and trapped electrons nonlinearly in the presence of ion and electron temperature gradients. The spectral intensity of the density fluctuations in the nonlinearly saturated state is analytically obtained from the steady state solution of the wave-kinetic equation. Distant nonlinear interactions between $l o w-k_{\theta}$ and high- $\mathrm{k}_{\theta}$ modes of similar frequencies via trapped electron scattering (the resonance between the beat wave and the trapped electron precession drift frequencies) suppress the low- $k_{\theta}\left[k_{\theta} \rho_{s} \ll\left(L_{n} / R\right)^{1 / 2}\right]$ modes while close interactions via ion Compton scattering (nonlinear ion Landau damping) produce a monotonically decreasing spectrum from $k_{\theta} \rho_{s} \cong\left(L_{n} / R\right)^{1 / 2}$ to $k_{\theta} \rho_{s} \cong 1$ according to an approximate power law $k_{\theta}{ }^{-3}$. Various fluctuation amplitudes at saturation and the fluctuation-induced anomalous particle and heat fluxes are found to be smaller than the mixing length estimates. The plasma confinement is predicted to improve with higher $T_{i} / T_{e}$, more peaked density profile, larger aspect ratio, and higher plasma current. Also, a significant dependence of transport on the electron temperature gradient is found which could coritribute to the rigidity of the electron temperature profile often experimentally observed.
\end{abstract}




\section{Introduction}

It is generally believed that low frequency microinstability-driven electrostatic turbulence plays an important role in determining the plasma transport in the interior region of tokamaks. 1,2 Indeed, transport models $3-5$ based on rather simple trapped electron drift wave turbulence pictures have performed relatively well in reproducing a number of tokamak confinement trends. For most of the present day large tokamak auxiliary heated plasmas, the collisionless trapped electron mode (CTEM) with $\omega_{\text {be }}>\omega, \omega_{* e}>\omega_{\text {de }}>v_{\text {eff }}$ rather than the dissipative trapped electron mode (DTEM) with $\omega_{\mathrm{be}}>v_{\mathrm{eff}}, \omega, \omega_{* \mathrm{e}}>\omega_{\text {de }}$ appears to be more relevant. 6,7 Here, $\omega$ is the mode frequency, $\omega_{* e}=C_{s} \rho_{s} k_{0} / L_{n}$ is the electron diamagnetic drift

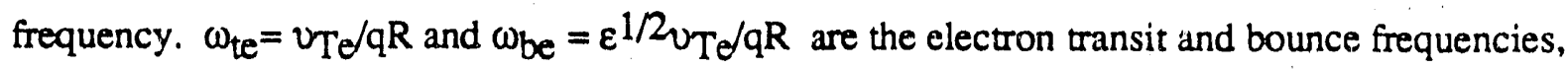
and $\omega_{\mathrm{de}}$ is the orbit-averaged trapped electron precession drift frequency.

The CTEM, which is destabilized by the trapped electron precession drift resonance has been analyzed extensively in the linear regime. ${ }^{8-10}$ The spatial structure of the most unstable eigenmode has been found to be localized in the bad curvature side of the tokamak with the parallel wavelength characterized by the connnection length. 11 This is true for a wide range of the poloidal wavelengths. 12 Since the mode is dispersive in the linearly unstable range, a number of weak turbulence approaches have been attempted based upon the wave-particle-wave nonlinear interactions. Most of these studies have been carried out within the context of local theory 13 or in a slab geometry. $14-15$

Chen et al., 13 have considered both the ion nonlinearity and the trapped electron nonlinearity in a slightly different collisionality regime; i.e., weakly dissipative trapped electron (WDTE) regime where $\omega_{\text {be }}>\omega, \omega_{* e}>v_{\text {eff }}>\omega_{\text {de }}$. The dominant nonlinearity in this case has been found to be the trapped electron nonlinearity which transfers the fluctuation energy to shorter wavelengths. Recently, Gang et al., 15 have developed a weak turbulence theory of CTEM in a sheared slab geometry in the absence of temperature gradients assuming the Pearlstein-Berk-type eigenmode structure. 16 They found that the trapped electron nonlinearity 
becomes weaker and that the ion Compton scattering, which transfers fluctuation energy to longer wavelengths, is the dominant nonlinear mechanism. The main reason for the different conclusions of these two previous studies is the difference in the eigenmode structure incorporated in the analyses; i.e., a local theory 13 vs. Pearlstein-Berk-type eigenmode structure ${ }^{15}$ in a sheared slab geometry. The ion Compton scattering has also been found to be the dominant nonlinear saturation mechanism for strongly dissipative trapped electron mode (SDTEM) with $\omega_{\text {be }}>v_{\text {eff }}>\omega, \omega_{* e}>\omega_{\text {de }}$ for sheared slab 14 and toroidal geometry 17 where the trapped electron dynamics can be treated linearly.

In this work, we develop a weak turbulence theory of CTEM in toroidal geometry in the presence of both ion and electron temperature gradients. We consider a simple model representation of the toroidal eigenmode structure which is found to be the most unstable form over a wide range of poloidal wavelengths. Ion Compton scattering and the trapped electron scattering are treated on an equal footing. The spectral intensity of the density fluctuation in the nonlinearly saturated state is analytically obtained from the steady state solution of the wave-kinetic equation. It is found that both nonlinearities are important in determining the spectral shape of the saturated fluctuations. Distant nonlinear interactions between low- $k_{\theta}$ and high- $\mathrm{k}_{\theta}$ modes of similar frequencies via trapped electron scattering (the resonance between the beat wave and the trapped electron precession drift frequencies) suppress the low- $k_{\theta}\left[k_{\theta} \rho_{s} \ll<\right.$ $\left.\left(L_{n} / R\right)^{1 / 2}\right]$ modes while close interactions via ion Compton scattering (nonlinear ion Landau damping) produce a monotonically decreasing spectrum from $k_{\theta} \rho_{s} \equiv\left(L_{n} / R\right)^{1 / 2}$ to $k_{\theta} \rho_{s} \equiv 1$ according to an approximate power law $\mathrm{k}_{\theta}{ }^{-3}$. Fluctuation amplitude at saturation and the fluctuation-induced anomalous particle and heat fluxes are then obtained. They are found to be smaller than the mixing length estimates. The plasma confinement is predicted to improve with higher $T_{i} / T_{e}$, more peaked density profile, larger aspect ratio, and higher plasma current. These trends are in agreement with recent experimental observations from TFTR transport studies. 6 Also, a significant dependence of transport on the electron temperature gradient is found. This 
might be relevant to the rigidity of the electron temperature profiles 18 often experimentally observed. 19

The remainder of this paper is organized as follows. In Sec.II, the nonlinear wave-kinetic equation, which describes the evolution of the fluctuation spectral intensity, is derived from the nonlinear ion gyrokinetic equation and the bounce-averaged nonlinear trapped-electron drift kinetic equation. Here, the respective roles of ion Compton scattering and trapped-electron scattering are also discussed. The spectral intensity at saturation is obtained as a steady-state solution of the wave-kinetic equation in Sec. III, and the fluctuation-induced fluxes as well as the anomalous electron-ion heat exchange rate are calculated in Sec. IV. Finally, the implications of the principal results from the present studies for the tokamak confinement are discussed in Sec. V.

\section{Derivation of Nonlinear Wave-Kinetic Equation}

In this section, we derive a wave-kinetic equation which describes the nonlinear evolution of the fluctuation spectral intensity. Before presenting the derivation, we briefly discuss the linear properties of collisionless trapped electron modes (CTEM) in toroidal geometry. Since the focus of this paper is on nonlinear theory, we adopt a simple model which captures the essential features of these instabilities. Our approach is to incorporate the relevant results from previous linear theories in a simple context in order to allow a systematic and tractable development of a weak turbulence nonlinear analysis for the CTEM.

The theoretical model considered in this paper consists of the Boltzmann response for the untrapped electrons and the collisionless bounce-averaged drift-kinetic equation for trapped electrons. Ions are treated by the collisionless gyrokinetic equation. The relevant frequencies in the linear analysis are ordered as $\omega_{\mathrm{te}}>\omega_{\mathrm{be}}>\omega, \omega_{* \mathrm{e}}>\omega_{\mathrm{de}}>v_{\mathrm{eff}}$, and $\omega_{,} \omega_{* \mathrm{e}}>\omega_{\mathrm{di}}, \omega_{\mathrm{ti}}$. 
Here, $\omega_{\mathrm{ti}}=v_{\mathrm{Ti}} / \mathrm{qR}$ is the ion transit frequency, the ion magnetic drift frequency is $\omega_{\mathrm{di}}$, and the orbit-averaged trapped electron precession drift frępuency is $\omega_{d e}=\omega_{* e}\left(L_{n} E / R T e\right) G(S, \kappa)$, where $S=r d l n q / d r$ is the magnetic shear parameter, and $K$ is the pitch angle variable related to the azimuthal angle $\theta_{0}$ of the turning point of a trapped particle by $\kappa=\sin \theta_{0}$. For a concentric high aspect ratio model equilibrium, $\mathrm{G}(\mathrm{S}, \mathrm{K})=2 \mathrm{E} / \mathrm{K}-1+4 \mathrm{~S}\left(\mathrm{E} / \mathrm{K}+\mathrm{K}^{2}-1\right), 20$ where $\mathrm{E}=\mathrm{E}(\mathrm{K})$ and $\mathrm{K}=$ $\mathbf{K}(\boldsymbol{K})$ are the Elliptic functions. Note that for higher $\mathrm{q}$ (which leads naturally to higher shear) $\mathrm{G}$ increases. This trend is also true for more realistic toroidal equilibria. ${ }^{21}$ Since $\omega>\omega_{b i}$ (the trapped ion bounce frequency), trapped ion dynamics are ignored. By taking appropriate perturbed density moments for each species in the linear regime, the quasi-neutrality condition yields the following eigenmode equation,

$$
\begin{aligned}
& (1+\tau) \Phi_{\mathbf{k}}+\tau \int_{\mathrm{d}^{3}} \mathrm{v}\left(\mathrm{iL}_{\mathrm{ik}}\right)\left[\omega-\omega_{* \mathrm{i}}\left(1+\left(\mathrm{u}^{2}-3\right) \eta_{\mathrm{i}} / 2\right\}\right] \mathrm{F}_{\mathrm{m}^{\mathrm{J}}}{ }^{2} \Phi_{\mathbf{k}} \\
& +\int d^{3} v\left(L_{e k}\right)\left[\omega-\omega_{* e}\left(1+\left(E / T_{e}-3 / 2\right) \eta_{e}\right\}\right] F_{n} \Phi_{k}=0,
\end{aligned}
$$

where $\Phi_{\mathbf{k}} \equiv \mathrm{e} \phi_{\mathbf{k}} / \mathrm{T}_{\mathrm{e}}$, and $\mathrm{L}_{\mathrm{ek}}=\mathrm{i}\left(\omega-\omega_{\mathrm{de}}+\mathrm{i} 0^{+}\right)^{-1}$ and $\mathrm{L}_{\mathrm{i} \mathbf{k}}=\mathrm{i}\left(\omega-\omega_{\mathrm{di}}-\mathrm{k}_{\|} \mathrm{v}_{\mathrm{i} \|}+\mathrm{i} 0^{+}\right)^{-1}$ are the linear test particle propagators in a toroidal geometry with magnetic shear. Although we have adopted for simplicity notations which are familiar from the local theory, it should be remembered that some of these quantities are really operators. In general, the solution to Eq. (1) requires numerical analysis and there have been numerous papers on this subject. $8-10$ The following results are of particular importance and interest for our purpose of developing a weak turbulence theory of collisionless trapped electron modes in a toroidal geometry.

i) A local dispersion relation has been derived for CTEM driven unstable by wave-trapped-electron-precession resonances using a fluid approximation $\left(\omega>\omega_{\mathrm{ti}}\right)$ for ions. 8 This driving mechanism remains robust even when progressively complicated and realistic configurations are considere. $8-10$

ii) A consequence of toroidal geometry is that each Fourier eigenmode radial width for the most unstable mode is approximately given by the distance between the nearest neighboring rational surfaces, $W_{n}=1 / k_{\theta} S$, due to linear interactions between different poloidal harmonics. 22 
Consequently, magnetic shear-induced ion Landau damping (which could be significant in a sheared slab geometry ${ }^{16}$ ) is rendered ineffective for most of the $k_{\theta}$ range of our interest where $\omega$ $>\omega_{\text {ti }}$ is satisfied. $9,10,22$ Therefore, the local dispersion relation of Ref.8 (where ion Landau damping effects are ignored) is a reasonable approximation.

iii) Describing the nonlinear evolution of toroidal drift waves using the ballooning mode formalism is extremely complicated. 17 In this paper, we develop a nonlinear theory in a configuration space assuming a simple gaussian-shaped radial structure 23 with a mode-width characteristic of results obtained from numerical eigenmode studies. 11

In order to simply model the main $k_{\theta}$ spectral features in the present problem, we adopt the following approximate form of the linear dispersion relation generated by Eq. (1),

$$
\omega\left(k_{\theta}\right)=v^{*} k_{\theta} /\left(1+k_{\theta}^{2} / k_{M^{2}}\right),
$$

where $k_{M}{ }^{-2} \equiv \rho_{s}^{2} /\left\{1+\tau^{-1}\left(1+\eta_{i}\right)\right\}$, and $v^{*}=\omega_{* e} / k_{\theta}$. Note that for $k_{\theta}^{2} / k_{M^{2}}<<1$, this reduces to the usual small Larmor radius expansion procedure result, $\omega\left(k_{\theta}\right)=v^{*} k_{\theta}\left(1-k_{\theta}{ }^{2} k_{M}{ }^{2}\right)$ which Eq. (2) successfully approximates for $\mathrm{k}_{\theta} 2 / \mathrm{k}_{\mathrm{M}}{ }^{2}<1$. The advantage of adopting the form of the dispersion relation given in Eq. (2) is that it makes the analysis of the nonlinear distant interaction of low $k$ and high $k$ modes much simpler. Our major conclusions are not affected by the approximate nature of this dispersion relation since most of the fluctuation energy exists in the long wave length range, $k_{\theta}^{2} / k_{M}{ }^{2} \ll 1$. This will be demonstrated in the next section. With regard to the destabilizing mechanism, the linear growth rate is assumed to be solely due to trapped electron precession resonances. From Ref. 8,

$$
\gamma \omega_{* e}=2(2 \pi \varepsilon)^{1 / 2}\left(R / G L_{n}\right)^{3 / 2} \exp \left(-R \Omega / G L_{n}\right) \Omega^{1 / 2}\left\{1-\Omega+\eta_{e}\left(R \Omega / G_{n}-3 / 2\right)\right\},
$$

where $\Omega=1 /\left(1+k_{\theta}^{2} / \mathrm{k}_{M}{ }^{2}\right)$.

Other nonresonant type driving mechanisms $24-26$ are not considered here since their nonlinear behaviors are not readily amenable to the weak turbulence approach 27 pursued in the present study. Finally, the radial mode structure is taken to be of the form,

$$
\left|\Phi_{k}(x)\right|^{2}=\left|\Phi_{k}(0)\right|^{2} \exp \left(-x^{2} / w_{n}^{2}\right)
$$


with $\mathrm{W}_{\mathrm{n}}=1 / \mathrm{k}_{\theta} \mathrm{S}$ being the approximate distance between the nearest neighboring rational surfaces.

Equations (2)-(4) summarize the linear properties of CTEM which are used in the following nonlinear calculations. Both ion and trapped electron nonlinearities are treated on an equal footing in the analysis. The trapped electron dynamics are given by the bounce-averaged nonlinear drift-kinetic equation,

$$
\left(\partial_{p}+i \omega_{d e}\right) h-i\left[\omega-\omega_{* e}\left(1+\left(E / T_{e}-3 / 2\right) \eta_{e}\right)\right]\langle\Phi\rangle F_{m}=\nabla\langle\Phi) \times \mathbf{b} \cdot \nabla h,
$$

where $h$ is the nonadiabatic part of the perturbed distribution function of trapped electrons; i.e., $f_{e l}=h+F_{m} e \phi / T_{e}, F_{m}=n_{0}\left(m_{e} / 2 \pi T_{e}\right)^{3 / 2} \exp \left(-E / T_{e}\right), \quad b=B / B \mid,\langle\Phi\rangle$ is the bounce-averaged value of $\Phi=e \phi / T_{e}$, and $\phi$ is the electrostatic potential. The untrapped electron dynamics can be approximated by the Boltzmann response as stated before. The collisionless nonlinear gyrokinetic equation 28 for ions is

$$
\left(j_{\mathrm{f}}+\mathrm{i} \omega_{\mathrm{di}}+v_{\|} \mathrm{b} \cdot \nabla\right) \mathrm{g}+\mathrm{i} \tau\left[\omega-\omega_{* \mathrm{i}}\left(1+\left(\mathrm{u}^{2}-3\right) \eta_{\mathrm{i}} / 2\right\}\right] \Phi J_{0} F_{\mathrm{m}}=\nabla \Phi J_{0} \times \mathbf{b} \cdot \nabla \mathrm{g},
$$

where $g$ is the nonadiabatic part of the perturbed distribution function; i.e., $g=f_{i 1}+F_{m} e \phi / T_{i}$, $F_{m}=n_{0}\left(M_{i} / 2 \pi T_{i}\right)^{3 / 2} \exp \left(-u^{2} / 2\right), u^{2}=M_{i} v^{2} / T_{i}$, and $J_{0}\left(k_{\perp} \rho_{i}\right)$ is a Bessel function.

We begin our analysis of Eq. (6) by noting that the right side represents the $E \times B$ convective nonlinearity. This term is assumed to be smaller than the linear terms in weak turbulence theory, i.e., $\Phi^{2}$ is on the order of $\gamma / \delta \omega<1$, where $\delta \omega=\omega_{* e}-\omega$. To the dominant order, the test mode at $\mathbf{k}$ obeys the linear relation.

$$
\begin{aligned}
& \mathrm{L}_{\text {ek }}{ }^{-1} \mathrm{~h}_{\mathbf{k}}(1)=\mathrm{i}\left[\omega-\omega_{* \mathrm{e}}\left\{1+(\mathrm{E}-3 / 2) \eta_{\mathrm{e}}\right\}\right]\langle\Phi\rangle_{\mathbf{k}} \mathrm{F}_{\mathrm{m}}, \\
& \mathrm{L}_{\mathrm{ik}}{ }^{-1} \mathrm{~g}_{\mathbf{k}}(1)=-\mathrm{i} \tau\left[\omega-\omega_{* \mathrm{i}}\left\{1+\left(\mathrm{u}^{2}-3\right) \eta_{\mathrm{i}} / 2\right\}\right] \mathrm{J}_{0} \Phi_{\mathbf{k}} \mathrm{F}_{\mathrm{m}},
\end{aligned}
$$

where the linear ion propagator has been simplified to $\mathrm{L}_{\mathrm{ik}}=\mathrm{i}\left(\omega-\mathrm{k}_{\|} \mathrm{v}_{\mathrm{i} \mid{ }^{+} \mathrm{i}^{+}}\right)^{-1}$ ignoring $\omega_{\mathrm{di}}$ compared to $k_{\|} v_{\|}$. The primary role of $\omega_{\text {di }}$ is to help set up a toroidal drift mode structure with $\mathrm{W}_{\mathrm{n}} \cong 1 / \mathrm{k}_{\theta} \mathrm{S}$ by introducing linear coupling between neighboring polöidal harmonics. 22 Trajıped electron dynamics can also contribute to this coupling. 29,30 The influence of $\omega_{\text {di }}$ on the growth rate is not appreciable and its influence on the downward shift of the real frequency is smaller 
than the finite gyroradius effects for $k_{\theta}^{2} / k_{M}{ }^{2}>L_{n} / R$. Since the radial mode width is on the order of $W_{n}$ for toroidal drift-wave, $\langle\Phi\rangle_{k} \equiv \Phi_{k}$. It is worthwhile to note that the bounce-average reduction $\left(\langle\Phi\rangle_{k}<\Phi_{k}\right)$ tends to be more significant for the Pearlstein-Berk-type modes which have characteristically broader radial widths. 15

To the next order, the mode at $\mathbf{k}^{\prime \prime}$ is driven by the nonlinear interaction of the test mode at $\mathbf{k}$ and the background modes at $\mathbf{k}^{\prime}$; i.e.,

$$
\begin{aligned}
& \mathrm{L}_{\text {ek }}{ }^{\prime-1} \mathrm{~h}_{\mathbf{k}^{\prime \prime}}{ }^{(2)}-\nabla \Phi_{\mathbf{k}} \times \mathbf{b} \cdot \nabla \mathrm{h}_{\mathbf{k}^{\prime}}(1)^{*}-\nabla \Phi_{\mathbf{k}^{\prime}}{ }^{*} \times \mathbf{b} \cdot \nabla \mathrm{h}_{\mathbf{k}}{ }^{(1)}=0, \\
& \mathrm{~L}_{\mathbf{i} \mathbf{k}^{\prime \prime}}{ }^{-1} \mathrm{~g}_{\mathbf{k}^{\prime \prime}}(2)-\nabla \Phi_{\mathbf{k}} \mathrm{J}_{0} \times \mathbf{b} \cdot \nabla \mathrm{g}_{\mathbf{k}^{\prime}}{ }^{(1) *}-\nabla \Phi_{\mathbf{k}^{\prime}}{ }^{*} \mathrm{~J}_{0^{\prime}} \times \mathbf{b} \cdot \nabla \mathrm{g}_{\mathbf{k}}(1)=0 \text {, }
\end{aligned}
$$

where $k^{\prime \prime}=\mathbf{k}-\mathbf{k}^{\prime}$, and $\langle\Phi\rangle_{\mathbf{k}}$ has been approximated by $\Phi_{\mathbf{k}}$. The "induced potential" $\Phi_{\mathbf{k}}{ }^{\prime \prime}(2)$ is obtained by imposing the quasi-neutrality condition at the second order.

$$
\begin{aligned}
& \varepsilon^{(1)}\left(\omega^{\prime \prime}, \mathbf{k}^{\prime \prime}\right) \Phi_{\mathbf{k}^{\prime \prime}}(2)=-i\left\{\left(\omega_{*} \mathrm{e}^{/ \omega_{\mathbf{k}}}\right)-\left(\omega * \mathrm{e}^{\left.\left.1 / \omega_{\mathbf{k}^{\prime}}\right)\right\}}\right.\right. \\
& {\left[\int_{d^{3}} 3 v\left(\omega^{\prime \prime}-k_{\|}{ }^{\prime \prime} v_{\|}+0^{+}\right)^{-1}\left[\mathrm{~J}_{0} J_{0} J_{0} "\left(1+\left(u^{2}-3\right) \eta_{i} / 2\right\}\right] F_{m}\right.} \\
& \left.-\int \mathrm{d} E E^{1 / 2}\left(\omega^{\prime \prime}-\omega_{\mathrm{de}}{ }^{\prime \prime}+\mathrm{i} 0^{+}\right)^{-1}\left\{1+(\mathrm{E}-3 / 2) \eta_{\mathrm{e}}\right\} \mathrm{F}_{\mathrm{m}}\right]\left(\mathrm{C}_{\mathrm{s}} \rho_{\mathrm{s}} \mathbf{k} \times \mathbf{k}^{\prime} \cdot \mathbf{b}\right) \Phi_{\mathbf{k}} \Phi_{\mathbf{k}^{\prime}} \text {, }
\end{aligned}
$$

where $J_{0}{ }^{\prime}=J_{0}\left(k_{\perp}^{\prime} \rho_{i}\right), J_{0} "=J_{0}\left(k^{\prime \prime} \rho_{i}\right), E$ has been normalized to $T_{e}$, and

$$
\begin{aligned}
& \varepsilon^{(1)}\left(\omega^{\prime \prime}, \mathbf{k}^{\prime \prime}\right) \phi_{\mathbf{k}} \equiv(1+\tau) \Phi_{\mathbf{k}} \\
& +\tau \int_{d^{3}} v\left(i L_{i k}{ }^{\prime \prime}\right) J_{0} 2\left[\omega^{\prime \prime}-\omega_{* i} "\left(1+\left(u^{2}-3\right) \eta_{i} / 2\right\}\right] F_{m} \Phi_{k} " \\
& +\int \mathrm{d}^{3} \mathrm{v}\left(\mathrm{iL} \mathrm{L}_{\mathrm{ek}}\right)\left[\omega^{\prime \prime}-\omega_{*} \mathrm{e}^{\prime \prime}\left(1+\left(\mathrm{E} / \mathrm{T}_{\mathrm{e}}-3 / 2\right) \eta_{\mathrm{e}}\right]\right] \mathrm{F}_{\mathrm{m}} \Phi_{\mathbf{k}^{\prime \prime}} \text {, }
\end{aligned}
$$

is the expression for the linear dielectric function. It is important to remember here that $\left(\omega ", \mathbf{b}_{\mathrm{b}}^{\prime \prime}\right)$ does not satisfy a linear dispersion relation $\varepsilon^{(1)}\left(\omega^{\prime \prime}, \mathbf{k}^{\prime \prime}\right)=0$, and that $\Phi_{\mathbf{k}}$ " is not a linear eigenmode. Furthermore, the assumed frequency ordering for the linear eigenmode is no longer valid; i.e., $\omega^{\prime \prime}<\omega_{\mathrm{cle}} ", \mathrm{k}_{\|}{ }^{\prime \prime} v_{\mathrm{i} \|}$. Consequently, both trapped electron and ion responses are weighted heavily by the contribution from the kinetic regime, and $\varepsilon^{(1)}\left(\omega^{\prime \prime}, \mathbf{k}^{\prime \prime}\right)$ deviates significantly from the local expression. The induced potential $\Phi_{\mathbf{k}}{ }^{\prime \prime}(2)$ is accordingly quite small, and the nonlinear shielding contribution is subdominant to the "bare" scattering terms. Of course, in the local approximation 13,31 or in the situation where the ion Landau damping is ignorable, 32 significant cancellation occurs between the shielding contribution and the "bare" scattering 
contributions at long wavelength.

To the third order, the back reaction of the driven modes on the test mode gives the nonlinear correction to the distribution function at $\mathbf{k}$;

$$
\begin{gathered}
L_{e k}{ }^{-1} h_{k^{(3)}}=-\Sigma_{k^{\prime}}\left(C_{s} \rho_{s} k \times k^{\prime} \cdot b\right)^{2}\left\{\Phi_{k^{\prime}}\left(h_{k^{\prime \prime}}(2)-i L_{e} k^{\prime \prime}\left[\omega^{\prime \prime}-\omega_{* e} "\left(1+\left(E / T_{e}-3 / 2\right) \eta_{e}\right\}\right] \Phi_{k^{\prime \prime}}\right)\right. \\
\left.-\Phi_{k^{\prime \prime}}(2)_{h_{k^{\prime}}}(1)\right\},
\end{gathered}
$$

and

$$
\begin{aligned}
& L_{i k}{ }^{-1} g_{k}(3)=-\Sigma_{k^{\prime}}\left(C_{s} \rho_{s} k \times k^{\prime} \cdot b\right)^{2}\left(J_{0}{ }^{\prime} \Phi_{k^{\prime}}\left(g_{k^{\prime \prime}}(2)-i \tau L_{k}{ }^{\prime \prime}\left[\omega^{\prime \prime}-\omega_{* i}{ }^{\prime \prime}\left(1+\left(u^{2}-3\right) \eta_{i} / 2\right\}\right] \Phi_{\mathbf{k}^{\prime \prime}}\right)\right. \\
& \left.-\mathrm{J}_{0}{ }^{\prime \prime} \Phi_{\mathbf{k}^{\prime \prime}}{ }^{(2)} \mathbf{g}_{\mathbf{k}^{\prime}}{ }^{(1)}\right\} \text {. }
\end{aligned}
$$

Here, we have retained only the "coherent" response $g_{k}(3)$ which is proportional to $\Phi_{k}$ by construction. Other "incoherent" contributions, which are not proportional to $\Phi_{k}$, are ignored. Although within this "coherent approximation" the fluctuation energy is no longer strictly conserved, we can nevertheless obtain useful information about the shape of the fluctuation k-spectrum. In Eqs. (12) and (13), the first term on the right side will hereafter be referred to as the "bare" contribution because this distribution function response is directly driven through the nonlinear interaction of $\Phi_{\mathbf{k}}$ with $\Phi_{\mathbf{k}^{\prime}}$ as described by Eqs. (8) and (9). The remaining two terms will be referred to as the "shielding" contribution since these are due to the $\Phi_{\mathbf{k}}$ "(2) fluctuation self-consistently induced by $g_{k}{ }^{\prime \prime}(2)$ and $h_{k^{\prime \prime}}(2)$. Finally, by requiring quasi-neutrality up to third order, we obtain the formal nonlinear eigenmode equation

$$
\varepsilon^{(1)}(\omega, k) \Phi_{k}=-\int d^{3} v h_{k}{ }^{(3)}+\int d^{3} v J_{0} g_{k}{ }^{(3)} \text {. }
$$

Here, the left hand side is the linear response; i.e.,

$$
\begin{aligned}
\varepsilon^{(1)}(\omega, \mathbf{k}) \Phi_{k} & =(1+\tau) \Phi_{k} \\
& +\tau \int_{d} 3 v\left(i L_{i k}\right)\left[\omega-\omega_{* i}\left(1+\left(u^{2}-3\right) \eta_{i} / 2\right)\right] F_{m} J_{0} \Phi_{k} \\
& +\int_{d} 3 v\left(i L_{e k}\right)\left[\omega-\omega_{* e}\left(1+\left(E / T_{e}-3 / 2\right) \eta_{e}\right)\right] F_{m} \Phi_{k}
\end{aligned}
$$

which is approximately equivalent to Eqs, (1)-(3) based on the frequency ordering for the linearly unstable range, $L_{n} / R<k_{0} / k_{M}<1$. At very short and very long wavelengths outside of this $k_{\theta}$ range, modes are linearly damped by ion Landau damping since the frequency becomes 
as low as the ion transit frequency. The first two terms on the right hand side of Eq. (15) are from the adiabatic responses of electrons and ions, respectively. The third term is the nonadiabatic ion linear response, and the last term represents the trapped electron response.

The right hand side of Eq. (14) contains the trapped electron nonlinear response from the trapped electron "bare" scattering and the nonlinear ion response from the ("bare") ion Compton scattering.

$$
-\int_{d^{3}} v_{k_{k}}(3)=\Sigma_{k^{\prime}}\left(C_{s} \rho_{s} k \times k^{\prime} \cdot \mathbf{b}\right)^{2}\left\langle L_{e k} L_{e k}{ }^{\prime \prime}\left(\left(a_{*} e^{/ \omega_{k}}\right)-\left(\omega * e^{\prime} / \omega_{k^{\prime}}\right)\right\}\left\{1+\left(E / T_{e}-3 / 2\right) \eta_{e}\right\}\right.
$$
\rangle$\left|\Phi_{\mathbf{k}}\right|^{2} \Phi_{\mathbf{k}}$

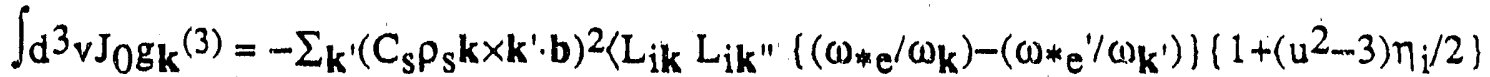

$$
\begin{aligned}
& \left.\mathrm{J}_{0}^{2} \mathrm{~J}_{0}{ }^{2}\right\rangle\left|\Phi_{\mathbf{k}^{\prime}}\right|^{2} \Phi_{\mathbf{k}}
\end{aligned}
$$

Here we have kept only the resonances from the (driven) beat mode particle interactions,

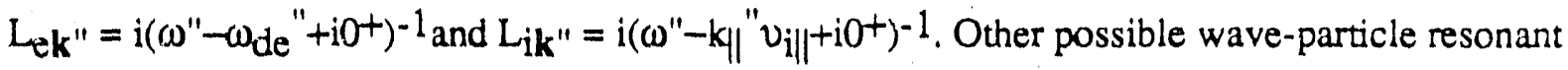
interactions at $\mathbf{k}$ or $\mathbf{k}^{\prime}$ are neglected in the nonlinear terms since only the high energy 'trapped electrons or high energy ions participate. The real part of Eq. (14) determines the eigenmode structure and the real frequency. By multiplying Eq. (14) with $\Phi_{\mathbf{k}^{*}}$ and taking the imaginary part, we obtain the wave-kinetic equation which describes the time evolution of the spectral intensity. The wave-kinetic equation obtained from Eq. (14) is,

$$
\begin{aligned}
& \left\{1 / 2\left(\partial \operatorname{Re} \varepsilon^{(1)} / \partial \omega\right) \partial / \partial t+\operatorname{Im} \varepsilon^{(1)}\right\}\left|\Phi_{k}\right|^{2}
\end{aligned}
$$

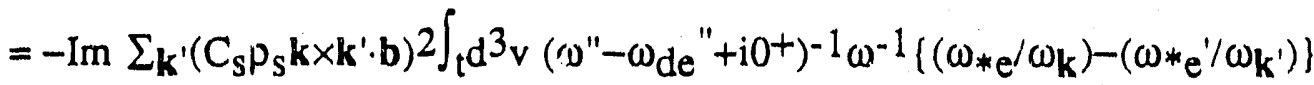

$$
\begin{aligned}
& \left\{1+\left(E / T e^{\left.-3 / 2) \eta_{e}\right\} F_{m}\left|\Phi_{k^{\prime}}\right| 2\left|\Phi_{k}\right|^{2}}\right.\right. \\
& +\operatorname{Im} \Sigma_{\mathbf{k}^{\prime}}\left(\mathrm{C}_{\mathrm{s}} \rho_{\mathrm{s}} \mathbf{k} \times \mathbf{k}^{\prime} \cdot \mathbf{b}\right)^{2} \int_{\mathrm{d}^{3}}{ }_{\mathrm{v}}\left(\omega^{\prime \prime}-\mathrm{k}_{\|} \|^{\prime \prime} v_{\mathrm{i} \|}+\mathrm{i} 0^{+}\right)^{-1} \omega^{-1}\left\{\left(\omega_{*} \mathrm{e}^{/ \omega_{k}}\right)-\left(\omega *^{\prime} \mathrm{e}^{\left.\left.1 / \omega_{\mathbf{k}^{\prime}}\right)\right\}}\right.\right. \\
& \left\{1+\left(\mathrm{u}^{2}-3\right) \eta_{\mathrm{i}} / 2\right\} \mathrm{J}_{0}{ }^{2} \mathrm{~J}_{0}{ }^{2} \mathrm{~F}_{\mathrm{m}}\left|\Phi_{\mathbf{k}}\right|^{2}\left|\Phi_{\mathbf{k}}\right|^{2}
\end{aligned}
$$

where the expression on the right hand side can be factored into a form,

$$
\left.M^{T E} S_{k k^{\prime}}\left|\Phi_{k^{\prime} \mid}\right| \Phi_{k}\right|^{2}+M^{I C S} S_{k k^{\prime}}\left|\Phi_{k^{\prime}}\right|^{2}\left|\Phi_{k}\right|^{2} \text {. For } k_{\theta}<k_{\theta}^{\prime} \text {, we can show that }
$$
$\mathrm{MTES}_{\mathbf{k k}^{\prime}}<0$ and $\mathrm{M}^{\mathrm{ICS}} \mathbf{k k}_{\mathbf{k}^{\prime}}>0$; i.e., trapped electron scattering $\left(\mathrm{M}^{\mathrm{TES}} \mathrm{kk}_{\mathbf{k}}\right)$ transfers 
fluctuation energy to the shorter wavelength modes while ion Compton scattering $\left(\mathrm{MICS}_{\mathbf{k k}} \mathbf{k}^{\prime}\right.$ transfers fluctuation energy to the longer wavelength modes in agreement with the local theory prediction. 13

In analytically performing the mode summation for various nonlinear interaction channels (i.e., the summation over all the background modes which satisfy $k^{\prime}=k-k^{\prime \prime}$, we adopt the following continuum approximation. 33 By changing the independent variables from $n^{\prime}, m^{\prime}$ to $k_{\phi}{ }^{\prime}$ and $x^{\prime}, \Sigma_{k^{\prime}}=\Sigma_{n^{\prime}, m^{\prime}} \cong \int d n^{\prime} d m^{\prime}=\int\left|\partial\left(n^{\prime}, m^{\prime}\right) / \partial\left(k_{\phi^{\prime}}, x^{\prime}\right)\right| d k_{\phi} d x^{\prime}=R^{2}(d q / d r) \int d k_{\phi^{\prime}} \mid k_{\phi^{\prime}} \int d x^{\prime} \cong$ $(\mathrm{rS} / \mathrm{q}) \int d \mathrm{k}_{\theta^{\prime}}\left|\mathrm{k}_{\theta^{\prime}}\right| \int_{d x}$.

We will also consider only a radial-mode-width averaged spectral intensity in order to make the wave-kinetic equation analytically tractable; i.e., we assume $\left|\Phi_{k}\right|^{2}=I\left(k_{\theta}\right) \exp \left(-x^{2} / w_{n}{ }^{2}\right)$, where $I_{\mathbf{k}}=I\left(\mathrm{k}_{\theta}\right)$ is the (radial-mode-width averaged) spectral intensity. Consequently, the right hand side can be evaluated explicitly at the rational surface of the test mode $(x=0)$ after using the appropriate radially nonlocal expreșsions, $\left(\mathbf{k} \times \mathbf{k}^{\prime} \cdot \mathbf{b}\right)^{2}=k_{\theta}{ }^{\prime 2} x^{\prime 2} / w_{n^{\prime}}{ }^{4}$, and $k_{\|}{ }^{\prime \prime} v_{\|}=k_{\theta}{ }^{\prime} x^{\prime} v_{\|} / L_{s}$. Then, the wave-kinetic equation simplifies to

$\left\{1 / 2(\partial \operatorname{Re\varepsilon }(1) / \partial \omega) \partial / \partial t+\operatorname{Im} \varepsilon^{(1)}\right\} I_{k}=$

$-\left(C_{s} \rho_{S}\right)^{2}(r S / q) \int d k_{\theta^{\prime}} \mid k_{\theta^{\prime}} \int d x^{\prime}\left(k_{\theta}{ }^{2} x^{\prime 2 / w_{n}}{ }^{4}\right) \exp \left(-x^{\prime 2} / w_{n^{\prime}}{ }^{2}\right) w_{k}{ }^{-1}$

$\left\{\left(\omega_{* e} / \omega_{k}\right)-\left(\omega * e^{\left.1 / \omega_{k}\right)}\right) \operatorname{Im} \int_{t^{d}}{ }^{3} v\left(\omega^{\prime \prime}-\omega_{d e} e^{\prime}+i 0^{+}\right)^{-1}\left(1+\left(E / T_{e}-3 / 2\right) \eta_{e}\right\} F_{m} I_{k^{\prime}} I_{k}\right.$

$+\left(C_{s} \rho_{s}\right)^{2}(r S / q) \int d k_{\theta^{\prime}} \mid k_{\theta^{\prime}} \int d x^{\prime}\left(k_{\theta^{\prime}} x^{\prime 2} / w_{n^{\prime}}{ }^{4}\right) \exp \left(-x^{\prime 2} / w_{n^{\prime}}{ }^{2}\right) \omega_{k^{-1}}$

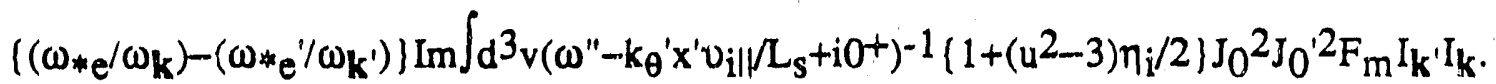

The dominant contribution to trapped electron scattering comes from the "distant" $k$-space interactions which are due to the beating between low $\mathrm{k}_{\theta}$ and high $\mathrm{k}_{\theta}^{\prime}$ fluctuations with similar frequencies; i.e., $\omega-\omega^{\prime} \ll<\omega \equiv \omega^{\prime}$, but $\omega_{*}<<\omega^{\prime} *_{e}$. The "close" $k$-space interactions between similar wavelength fluctuations are weak due to the fact that $\omega_{\text {de }}^{\prime}-\omega_{\text {de }} \ll<\omega^{\prime}-\omega$, since $\omega_{\text {de }} \ll$ $\omega$ and $\omega_{\mathrm{de}}{ }^{\prime}<<\omega^{\prime}$. Therefore, we keep only the distant interaction contributions for the $k_{\theta}{ }^{\prime}$ sum. To facilitate the explicit evaluation of the distant interactions, we assume a specific $\mathrm{k}_{\theta}$-dependence of the linear frequency given in Eq. (2). This yields a simple relation between $\mathrm{k}_{\theta}$ 
and its short wavelength counterpart $k_{\theta}^{t}$ with the same frequency [i.e., $\omega\left(k_{\theta}\right)=\omega\left(k_{\theta}^{t}\right), k_{\theta} \neq k_{\theta}^{t}$

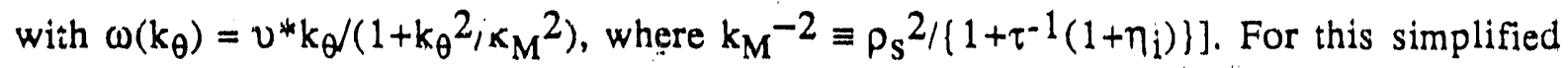
dispersion relation, the reciprocal relation for the distant interaction is $k_{\theta}^{t} / k_{M} \cong k_{M} / k_{\theta}$. We also note that this specific form of the dispersion relation does not affect our main conclusions about the long wavelength spectrum at saturation. For a given $\mathrm{k}_{\theta}$, the distant interaction occurs mainly with $\mathrm{k}_{\theta}^{\prime}$ such that $\omega^{\prime \prime} / \omega_{\mathrm{de}} \mathrm{e}^{\prime \prime}<1$. Therefore, the integration is simplified by expanding $\omega^{\prime \prime}$ around $k_{\theta}^{\prime} \cong k_{\theta}^{t} ;$ i.e., $\omega^{\prime \prime}=\omega\left(k_{\theta}\right)-\omega\left(k_{\theta}^{\prime}\right) \cong-\left(k_{\theta}^{\prime}-k_{\theta}^{t}\right)(\partial \omega / \partial p)_{k t}$, where we use $\omega(p)$ to indicate the $k_{\theta}$ dependence of $\omega$ and to avoid possible confusion with the test mode $k_{\theta}$. Also $I\left(k_{\theta}^{\prime}\right)$ is expanded around $k_{\theta}^{t}, I\left(k_{\theta}^{\prime}\right) \cong I\left(k_{\theta}^{t}\right)+\left(k_{\theta}^{\prime}-k_{\theta}^{t}\right)(\partial I(p) / \partial p)_{k t}$. The velocity space resonant integral and the radial integration over the background mode width are straightforward. The $\mathrm{k}_{\theta}^{\prime}$-integration is facilitated by changing the integration variable to $\omega^{\prime \prime} / \omega_{\mathrm{de}}$ " using $\omega^{\prime \prime} / \omega_{\mathrm{de}} " \equiv$ $-\left(k_{\theta}^{\prime}-k^{t}{ }_{\theta}\right)\left(k_{\theta}-k_{\theta}^{t}\right)^{-1}(\partial \omega / \partial p)_{k t}\left(e B R / c T_{e} G\right)$. Finally, the trapped electron scattering contribution [the first term on the right side of Eq. (19)] becomes

$$
\begin{aligned}
& \left.-(\pi / 2)\left(\mathrm{C}_{\mathrm{s}} \rho_{\mathrm{s}}\right)^{2}\left(\mathrm{rs} \mathrm{s}^{2} / \mathrm{q}\right)(2 \pi \varepsilon)^{1 / 2} \mathrm{k}_{\mathrm{M}^{2}} \mathrm{k}_{\theta}{ }^{2} \mathrm{k}_{\theta}^{\mathrm{t}}{ }^{2}\right) \omega\left(\mathrm{k}_{\theta}\right)^{-1} \mid \partial \omega / \partial \mathrm{pl} \mathrm{k}^{\mathrm{r}^{-1}} \\
& \left\{I\left(k_{\theta}^{t}\right)-3\left(1+\eta_{e}\right) 2^{-1} \omega^{\prime \prime} \operatorname{de}^{(\partial \omega / \partial p)} k^{t^{-1} \partial I / \partial p l} k^{t}\right\} \text {. }
\end{aligned}
$$

For the ion Compton scattering, the dominant contribution comes from the close interactions between modes with similar frequencies and similar $\mathbf{k}$ 's. This is because the distant interaction which involves high-k' contributions is reduced by the gyro-orbit average factor $\mathrm{J}_{0}{ }^{2}$. For close interactions between low $\mathbf{k}$ modes, we can approximate the Bessei functions by 1 . After carrying out the resonant velocity space integration, the ion Compton scattering term in Eq. (19) becomes

$$
\begin{aligned}
& \left(\mathrm{C}_{\mathrm{s}} \rho_{\mathrm{s}}\right)^{2}(\mathrm{rS} / \mathrm{q}) \int \mathrm{dk}_{\theta^{\prime}} \mid \mathrm{k}_{\theta^{\prime}}{ }^{\prime} \int \mathrm{dx}^{\prime} \mathrm{k}_{\theta^{\prime}}{ }^{2} \mathrm{x}^{\prime 2} \mathrm{w}_{\mathrm{n}^{\prime}}-4 \exp \left(-\mathrm{x}^{\prime 2} / \mathrm{w}_{\mathrm{n}^{\prime}}{ }^{2}\right) \omega_{\mathrm{k}}{ }^{-1} \\
& \left\{\left(\omega_{*} e / \omega_{\mathbf{k}}\right)-\left(\omega *_{e^{\prime}} / \omega_{\mathbf{k}^{\prime}}\right)\right\}(\pi / 2)^{1 / 2}\left|\mathbf{k}_{\theta}{ }^{\prime} x^{\prime} v_{t_{i}} / L_{s}\right|^{-1}\left\{1+\left(\left|k_{\theta^{\prime}} x^{\prime} v_{t_{i}} / \omega^{\prime \prime} L_{s}\right|-2-1\right) \eta_{i} / 2\right\} \\
& \exp \left\{-\left|\mathrm{k}_{\theta}{ }^{\prime} x^{\prime} v_{t i} / \omega " L_{s}\right|-2 / 2\right\} \mathrm{I}_{\mathbf{k}^{\prime} \mathbf{k}_{\mathbf{k}}} \text {. }
\end{aligned}
$$

The last exponential factor indicates that the ion Compton scattering amplitude increases with $k_{\|} "$ $\propto x^{\prime}$, while the extent of $x^{\prime}$ is limited by a radial mode structure factor, $\exp \left(-x^{\prime} / w_{n^{\prime}}{ }^{2}\right)$. Next, the radial integration, which accounts for a part of the mode summation, can be approximately 
evaluated by the saddle point method observing that the controlling factors are the two aforementioned exponential dependencies. The integration yields

$$
\begin{aligned}
& \left(\mathrm{C}_{\mathrm{s}} \rho_{\mathrm{s}}\right)^{2}(\mathrm{rS} / \mathrm{q}) \int d \mathrm{~d}_{\theta^{\prime}}\left|\mathrm{k}_{\theta}{ }^{\prime}\right| \mathrm{k}_{\theta}{ }^{\prime} \mathrm{w}_{\mathrm{n}^{\prime}}-4 \omega_{\mathrm{k}}-1
\end{aligned}
$$

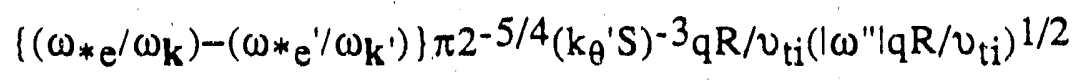

$$
\begin{aligned}
& \left\{1+\left(\sqrt{ } 2\left|\omega^{\prime \prime}\right| \mathrm{qR} / v_{\mathrm{t}^{-}}-1\right) \eta_{\mathrm{i}} / 2\right\} \exp \left\{-\sqrt{ } 2\left|\omega^{\prime \prime}\right| \mathrm{qR} / \nu_{\mathrm{ti}}\right\} I_{\mathbf{k}^{\prime}} \mathbf{I}_{\mathbf{k}} \text {. }
\end{aligned}
$$

The equation above clearly indicates that only the low frequency beat waves with $|\omega| \mid<v_{t i} / q R$ strusgly interact with the ion distribution function and contribute significantly to the spectrum evolution. We then perform the $k_{\theta}$ '-integration using the following differential approximation for close interactions, $27,33 \mathrm{I}\left(\mathrm{k}_{\theta}^{\prime}\right) \equiv \mathrm{I}\left(\mathrm{k}_{\theta}\right)+\left(\mathrm{k}_{\theta}^{\prime}-\mathrm{k}_{\theta}\right)(\partial \mathrm{I}(\mathrm{p}) / \partial \mathrm{p})_{\mathrm{k}}$. Finally combining with Eq. (20), the RHS of the wave-kinetic equation simplifies to

$$
\begin{aligned}
& 15 \pi^{3 / 2} 2^{-4}\left(C_{s} \rho_{s}\right)^{2}\left(r S^{2} / q\right)\left(v_{t i} / q R\right)^{2}\left(1+5 \eta_{i} / 4\right) k_{\theta}^{3} \omega\left(k_{\theta}\right)^{-1}\left|\partial \omega / \partial k_{\theta}\right|^{-3} k_{M^{-2}} \\
& \mathrm{I}\left(\mathrm{k}_{\theta}\right) \partial / \partial \mathrm{k}_{\theta}\left\{\mathrm{k}_{\theta} 2 \mathrm{I}\left(\mathrm{k}_{\theta}\right)\right\} \\
& -(\pi / 2)\left(\mathrm{C}_{\mathrm{s}} \rho_{\mathrm{S}}\right)^{2}(\mathrm{rs} / \mathrm{q})(2 \pi \varepsilon)^{1 / 2} \mathrm{k}_{\mathrm{M}}{ }^{2}\left(\mathrm{k}_{\theta}{ }^{2} \mathrm{k}_{\theta}^{\mathrm{t}}{ }^{2}\right) \omega\left(\mathrm{k}_{\theta}\right)^{-1} \mid \partial \omega / \partial \mathrm{pl} \mathrm{k}^{\mathrm{r}^{-1}} \\
& \left\{\left(k_{\theta}^{t}\right)-3\left(1+\eta_{e}\right) 2^{-1} \omega^{\prime \prime} d_{e}(\partial \omega / \partial p)_{k^{t}}-1 \partial U / \partial p_{k} t\right\} \text {. }
\end{aligned}
$$




\section{Fluctuation Spectrum at Saturation}

In the preceding section, we have derived a wave-kinetic equation which describes the nonlinear evolution of the fluctuation spectrum. In this section we solve that equation at nonlinear saturation. Since $\partial \mathrm{I}\left(\mathrm{k}_{\theta}\right) / \partial \mathrm{t}=0$ at saturation, we can write Eq. (23) in the following form to emphasize the $k_{\theta}$-dependences,

$$
\begin{aligned}
& -\beta L \mathrm{~L}\left(\Omega\left(\mathrm{k}_{\theta}\right)\right)= \\
& \beta^{I C S}{k_{\theta}}^{3} \omega\left(k_{\theta}\right)^{-1} \partial \omega \omega \partial k_{\theta} \mid-3 \partial\left\{k_{\theta}{ }^{2} I\left(k_{\theta}\right)\right\} / \partial k_{\theta}
\end{aligned}
$$

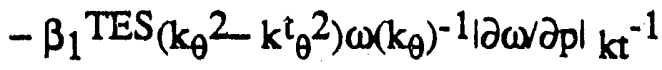

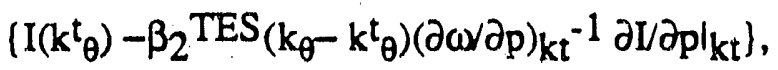

$$
\begin{aligned}
& \text { where } \beta^{L}=2(2 \pi \varepsilon)^{1 / 2}\left(R / G L_{n}\right)^{3 / 2}, \Omega=1 /\left(1+k_{\theta} 2 / k_{M}{ }^{2}\right) \text {, } \\
& F\left(\Omega\left(k_{\theta}\right)\right)=\exp \left(-R \Omega / G L_{n}\right) \Omega^{1 / 2}\left\{1-\Omega+\eta_{e}\left(R \Omega / G L_{n}-3 / 2\right)\right\} \text {, } \\
& \beta^{I C S}=15 \pi^{3 / 2} 2^{-4}\left(C_{s} \rho_{s}\right)^{2}\left(\mathrm{rS}^{2} / \mathrm{q}\right)\left(v_{\mathrm{ti}} / \mathrm{qR}\right)^{2}\left(1+5 \eta_{\mathrm{i}} / 4\right) \mathrm{k}_{\mathrm{M}^{-2}} \text {, } \\
& \beta_{1} \text { TES }=(\pi / 2)\left(C_{s} \rho_{s}\right)^{2}\left(r S^{2} / q\right)(2 \pi \varepsilon)^{1 / 2} \mathrm{k}_{M^{2}} \text {, } \\
& \beta_{2} \text { TES }=3\left(1+\eta_{e}\right) G L_{n}\left(C_{s} \rho_{s}\right) / 2 R \text {. }
\end{aligned}
$$

First, we consider wavelengths in the range, $k_{\theta}<k_{M}$, which are mainly responsible for the induced transport. Observing that the ion Compton scattering term in Eq. (24) is proportional to $\left|\partial \omega / \partial \mathrm{k}_{\theta}\right|^{-3}$ and that the trapped electron scattering term has a factor $\left(\mathrm{k}_{\theta}{ }^{2}\right.$ $\left.k^{t}{ }^{2}\right) \omega\left(k_{\theta}\right)^{-1} \mid \partial \omega / \partial p k t^{-1}$, we expect that as $k_{\theta} \Rightarrow k_{M}$ (then, $k_{\theta}^{t} \Rightarrow k_{M}$ ) the ion Compton scattering becomes stronger while the trapped electron scattering becomes weaker. Therefore, for $\mathrm{k}_{\mathrm{L}}<\mathrm{k}_{\theta}<\mathrm{k}_{\mathrm{M}}$, where $\mathrm{k}_{\mathrm{L}}$ is to be determined later, we can ignore the trapped electron scattering and get a spectrum at saturation by balancing the linear drive [the LHS of Eq. (24)] against the ion Compton scattering (the 1st term on the RHS). Then, Eq. (24) simplifies to the following first order inhomogeneous differential equation for the spectral intensity $I\left(k_{\theta}\right)$,

$$
\begin{aligned}
& -\beta L F\left(\Omega\left(k_{\theta}\right)\right)= \\
& \beta^{I C S}\left(C_{s} \rho_{S} / L_{n}\right)^{-4} k_{\theta}^{2}\left(1+k_{\theta}{ }^{2} / k_{M}{ }^{2}\right)^{7 \mid 1-k_{\theta}}{ }^{2} / k_{M}{ }^{21-3} \partial / \partial k_{\theta}\left\{k_{\theta}{ }^{2} I\left(k_{\theta}\right)\right\} .
\end{aligned}
$$


Taking an inhomogeneous solution which decays to zero as $\mathrm{k}_{\theta}$ becomes larger, we get

$$
I\left(k_{\theta}\right)=(\beta L / \beta I C S)\left(C_{S} \rho_{S} / L_{n}\right)^{4} k_{\theta}-2 \int_{k \theta} d k_{\theta} k_{\theta}{ }^{-2}\left(1+k_{\theta}{ }^{2} / k_{M}{ }^{2}\right)-7 / 1-k_{\theta} 2 / k_{M}{ }^{2 / 3} F(\Omega) .
$$

Here, we note that $I\left(k_{\theta}\right)$ vanishes as $k_{\theta} \Rightarrow k_{M}$. However, this is only an artifact of our approximate treatment of mode summation with $\omega^{\prime \prime} \cong-\left(k_{\theta}^{\prime}-k_{\theta}\right)(\partial \omega / \partial p)_{k}$. Since our approximation becomes poorer as $\mathrm{k}_{\theta} \Rightarrow \mathrm{k}_{\mathrm{M}}$, we cannot predict a detailed shape of the spectrum in that wavelength regime. Nevertheless, it is clear that $I\left(k_{\theta}\right)$ decays faster than $k_{\theta}{ }^{-3}$. For $k_{\theta}$ $\ll \mathrm{k}_{\mathrm{M}}$, Eq. (26) can be further simplified to

$$
\begin{aligned}
\mathrm{I}\left(\mathrm{k}_{\theta}\right) \cong & \left(2^{5}(2 \varepsilon)^{1 / 2} / 15 \pi\right)\left(\mathrm{q}^{3} / \mathrm{S}^{2}\right)\left(\mathrm{T}_{\theta} / \mathrm{T}_{\mathrm{i}}\right)\left(1+5 \eta_{\mathrm{i}} / 4\right)^{-1}\left(\mathrm{kMP}_{\mathrm{S}}\right)^{2}\left(\mathrm{R} / \mathrm{L}_{\mathrm{n}}\right)^{2}\left(\mathrm{R} / \mathrm{GL}_{\mathrm{n}}\right)^{3 / 2} \\
& \left(\mathrm{R} / \mathrm{GL}_{\mathrm{n}}-3 / 2\right) \exp \left(-\mathrm{R} / \mathrm{GL}_{\mathrm{n}}\right) \mathrm{r}^{-1} \mathrm{~L}_{\mathrm{Te}}{ }^{-1} \mathrm{~L}_{\mathrm{n}}{ }^{-1} \mathrm{k}_{\theta}{ }^{-3} .
\end{aligned}
$$

Next, we consider the range $k_{\theta}<k_{L}$, where the ion Compton scattering is negligible. Keeping only the linear term and the trapped electron scattering term from Eq. (24), we have

$$
\begin{aligned}
& -\beta^{L} F\left(\Omega\left(k_{\theta}\right)\right)= \\
& \left.-\beta_{1} \operatorname{TES}_{\left(k_{\theta}\right.}{ }^{2} k^{t}{ }_{\theta}^{2}\right) \omega\left(k_{\theta}\right)^{-1}|\partial \omega / \partial|_{k} t^{-1} \\
& \left\{I\left(k^{t}\right)-\beta_{2} \operatorname{TES}_{\left.\left(k_{\theta}-k^{t}\right)(\partial \omega / \partial p)_{k} t^{-1} \partial I / \partial p_{k}{ }^{t}\right\} .}\right.
\end{aligned}
$$

Since we want to obtain the spectrum in the very long wavelength regime, it is convenient to get a solution $I\left(k_{\theta}^{t}\right)$ for $k_{\theta}^{t}<k_{M}<k_{\theta}$. The right side of this equation can be simplified by introducing an integrating factor $S(\phi)$,

$$
\mathrm{dS} / \mathrm{d} \phi=\left(\beta^{\mathrm{L}} / \beta_{1} \mathrm{TES}_{\beta_{2}} \mathrm{TES}\right)\left(\mathrm{C}_{\mathrm{s}} \rho_{\mathrm{S}} / \mathrm{L}_{\mathrm{n}}\right)^{3} / \mathrm{k}_{\mathrm{M}} \exp \left(-\left(\mathrm{C}_{s} \rho_{\mathrm{S}} / 2 \mathrm{~L}_{\mathrm{n}} \beta_{2} \mathrm{TES}\right) \phi^{2}\right) \phi^{4} \mathrm{f}(\Omega),
$$

where $S(\phi)=\exp \left(-\left(C_{S} \rho_{S} / 2 L_{n} \beta_{2}{ }^{T E S}\right) \phi^{2}\right) I(\phi)$, and $\phi=k_{\theta}^{t} / k_{M}$. Since the trapped electron scattering transfers energy to shorter wavelengths, and the linear ion Landau damping becomes effective at very long wavelengths, we take a solution which vanishes as $k_{\theta} / k_{M} \Rightarrow 0$. Then, a simple integration yields

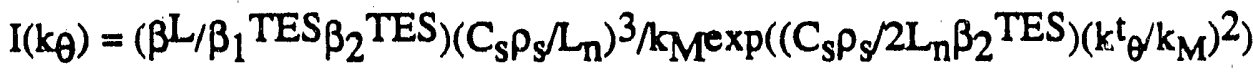

$$
\begin{aligned}
& \int \mathrm{k}_{\theta} \mathrm{d} \phi \mathrm{F}\left(\Omega\left(\mathrm{k}_{\theta}\right)\right) \exp \left(-\left(\mathrm{C}_{s} \rho_{s} / \mathrm{I}_{n} \beta_{2}{ }^{\mathrm{TES}}\right) \phi^{2}\right) \phi^{4} \text {. }
\end{aligned}
$$

Since $\phi<\mathrm{k}_{\theta} / \mathrm{k}_{\mathbf{M}}<<1$, the integral in Eq. (30) can be further simplified to 


$$
\int k t_{d \phi \exp }\left(-\left(1+1 / 3\left(1+\eta_{e}\right)\right) R \phi^{2} / G L_{n}\right) \phi^{5}\left(1+\eta_{e}\left(R \phi^{2} / G L_{n}-3 / 2\right)\right\} \text {. }
$$

If $\eta_{e}>2 / 3$, the lower limit of the integral should be modified to $\left\{\operatorname{GLTe}_{\mathrm{Te}}\left(3 \eta_{\mathrm{e}} / 2-1\right) / \mathrm{R}\right\}^{1 / 2}$. However, it will be shown later that in practice this detailed refinement is not really required. This is because the trapped electron nonlinear scattering makes the spectrum in this region very small, regardless of the cietailed treatment of the kinetic ion linear response. Effectively, the nonlinear trapped electron response determines the low-k spectrum cutoff. This is in contrast to most previous theories where either the three-wave decay 27 or the linear damping 15,18 has been responsible for the low-k spectrum cutoff. For $\left(k_{\theta} / k_{M}\right)^{2} \ll<L_{n} / R$, the integral increases very sharply with $k_{\theta}$ (according to $k_{\theta}^{8}$ ), although the magnitude stays very small. For $\left(k_{\sigma} / k_{M}\right)^{2}>L_{n} / R$, the integrand begins to decrease due to the exponential factor and the integral asymptotes to a constant value as $\mathrm{k}_{\theta}$ is increased. The constant is easily found to be $\mathrm{C}^{\mathrm{T}}=$ $\left(\mathrm{R}\left(4+3 \eta_{\mathrm{e}}\right) / \mathrm{L}_{\mathrm{n}}\left(3+3 \eta_{\mathrm{e}}\right)\right)^{-4}\left(\mathrm{R} / 2 \mathrm{~L}_{\mathrm{Te}}\right)\left\{\left(3 \eta_{\mathrm{e}}{ }^{2}+4 \eta_{\mathrm{e}}+8 / 3\right) /\left(\eta_{\mathrm{e}}{ }^{2}+\eta_{\mathrm{e}}\right)\right\}$. Thus, $\mathrm{I}\left(\mathrm{k}_{\theta}\right)$ is extremely small for $\left(k_{\theta} / k_{M}\right)^{2}<L_{n} / R$ and monotonically increasing. For $\left(k_{L} / k_{M}\right)^{2}>\left(k_{\theta} / k_{M}\right)^{2}>L_{n} / R$, $\mathrm{I}\left(\mathrm{k}_{\theta}\right)$ is approximately given by

$$
\mathrm{C}^{\mathrm{T}}\left(\mathrm{qR} / \mathrm{S}^{2} \mathrm{r}\right) \eta_{\mathrm{e}}\left(1+\eta_{\mathrm{e}}\right)^{-1}\left(\mathrm{k}_{\mathrm{M}^{\mathrm{L}}} \mathrm{L}^{-3}\left(\mathrm{R} / \mathrm{GL}_{\mathrm{n}}\right)^{-3 / 2} \exp \left(\left(\mathrm{C}_{\mathrm{s}} \rho_{\mathrm{S}} / 2 \mathrm{~L}_{\mathrm{n}} \beta_{2} \mathrm{TES}\right)\left(\mathrm{k}^{\mathrm{t}} \mathrm{k}_{\mathrm{M}}\right)^{2}\right)\right. \text {. }
$$
Since Eq. (26) predicts a monotonically decreasing $I\left(k_{\theta}\right)$ for $\mathrm{k}_{\mathrm{L}}<\mathrm{k}_{\theta}<\mathrm{k}_{\mathrm{M}}$, it is clear that the spectral intensity peaks at $\mathrm{k}_{\mathrm{L}}$. Now, we can determine $\mathrm{k}_{\mathrm{L}}$ by equating. Eq. (27) with Eq. (32) at $\mathrm{k}_{\theta}=\mathrm{k}_{\mathrm{L}}$. After some algebraic arrangement, we obtain

$$
\begin{gathered}
\left(\mathrm{kL}_{\mathrm{L}} / \mathrm{kM}^{3}\right)^{3} \exp \left[\left(\mathrm{R} / 3 \mathrm{GL}_{\mathrm{n}}\right)\left(1+\eta_{\mathrm{e}}\right)^{-1}\left(\mathrm{k}_{\mathrm{L}} / \mathrm{k}_{\mathrm{M}}\right)^{2}\right]=\left(2^{5}(2 \varepsilon)^{1 / 2} / 15 \pi\right) \mathrm{q}^{2}\left(\mathrm{~T}_{\mathrm{e}} / \mathrm{T}_{\mathrm{i}}\right) \eta_{\mathrm{e}}\left(1+\eta_{\mathrm{e}}\right)^{-1} \\
\left(1+5 \eta_{\mathrm{i}} / 4\right)^{-1}\left(\mathrm{kMP}_{\mathrm{S}}\right)^{2}\left(\mathrm{R} / \mathrm{L}_{\mathrm{n}}\right)\left(\mathrm{R} / \mathrm{GL}_{\mathrm{n}}\right)^{3}\left(\mathrm{R}_{\mathrm{GL}}-3 / 2\right) \exp \left(-\mathrm{R} / \mathrm{GL}_{\mathrm{n}}\right) / \mathrm{C}^{\mathrm{T}}
\end{gathered}
$$

Since Eq. (33) is a transcendental equation, it does not yield a simple formula for $\mathrm{k}_{\mathrm{L}}$. Nevertheless, considering typical parameters for large tokamaks, the right side of this equation is of order unity, and we have $\left(k_{L} / k_{M}\right)^{2}<3\left(1+\eta_{e}\right) G L_{n} / R$. The expected shape of the spectral intensity, $\mathrm{I}\left(\mathrm{k}_{\theta}\right)$, is plotted in Fig. 1 , signifying three differen: asymptotic regions in $\mathrm{k}_{\theta}$. The region " $a$ " corresponds to an extremely long wavelength regime where fluctuations are 
suppressed due to the trapped electron scattering. The region " $b$ " corresponds to a long wavelength regime where most of the fluctuation energy is populated and $I\left(k_{\theta}\right)$ decays according to a power law, $\mathrm{k}_{\theta}{ }^{-3}$. Finally, region " $\mathrm{c}$ " corresponds to a relatively shorter wavelength regime, $k_{\theta}>k_{M}$, where $I\left(k_{\theta}\right)$ decays more steeply. We note that the saturated amplitude of the fluctuations is smaller than the usual rough mixing length estimates, $I\left(k_{\theta}\right) \cong$ $1 / k_{\theta}{ }^{2}{ }_{n}^{2}$. Also, the spectral shape exhibits a $k_{\theta}$-dependence which is steeper than the mixing length prediction. These features are in rough agreement with most microwave scattering measurement results from tokamaks. $34-37$

\section{Fluctuation-induced Transport}

In this Section, we calculate the anomalous particle and thermal fluxes of both electrons and ions induced by the nonlinearly saturated trapped electron driven drift wave fluctuations. Since the particle transport obeys the ambipolarity constraint, it suffices to consider only the electron particle flux. The untrapped electron transport is very small because the drift wave fluctuation resonates only with the extremely low velocity part of the distrbution function, due to its slow phase velocity along the magnetic field line. Therefore, the trapped electrons are transported preferentially. Since the flux is induced by the radial component of the $E \times B$ velocity involving the fluctuating $E_{\theta}$ and the density fluctuation, the electron particle flux is given by

$$
\Gamma_{e}=\operatorname{Re} \Sigma_{k} i_{\theta} c T_{e} \Phi_{-k} \int d^{3} v\left(h_{k}(1)+h_{k}{ }^{(3)}\right) / e B_{0}
$$

First, we note that the contribution from $h_{k}(3)$ is proportional to $\operatorname{Im} \Sigma_{k^{\prime}}\left(C_{s} \rho_{s} k \times k^{\prime} \cdot b\right)^{2}$ $\int_{t} d^{3} v\left(\omega^{\prime \prime}-\omega_{d e}{ }^{\prime \prime}+i 0^{+}\right)^{-1} \omega^{-1}\left\{\left(\omega_{*} e^{/ \omega_{k}}\right)-\left(\omega_{*} e^{1 / \omega_{k}}\right)\right\}\left\{1+\left(E / T_{e}-3 / 2\right) \eta_{e}\right\} F_{m} I_{k^{\prime}} I_{k} ;$ i.e., approximately the $k$-sum of the product of the intensity at $k_{\theta}$ and the intensity at the conjugate 
wave number $\mathrm{k}_{\theta}^{\mathrm{t}}, \mathrm{I}\left(\mathrm{k}_{\theta}^{\mathrm{t}}\right)$. However, for low $\mathrm{k}_{\theta}, \mathrm{I}\left(\mathrm{k}_{\theta}^{\mathrm{t}}\right)$ is extremely small and vice-versa for high $k_{\theta}$. As a consequence, the product $I\left(k_{\theta}\right) I\left(k_{\theta}^{t}\right)$ is always small. Therefore, the dominant contribution is from $h_{k}(1)$, which can be written in an integral form using a continuum approximation and straight-forward velocity space integration; i.e.,

$$
\begin{aligned}
& \Gamma_{\mathrm{e}}=\operatorname{Re} \Sigma_{\mathbf{k}} \mathrm{ik}_{\theta}\left(\mathrm{cT}_{\mathrm{e}} / \mathrm{eB} \mathrm{B}_{0}\right) \Phi_{-k} \iint_{\mathrm{d}} 3_{\mathrm{v}} h_{\mathbf{k}}(1) \\
& =\left(\mathrm{cT}_{e} / \mathrm{eB}_{0}\right)(\mathrm{rS} / \mathrm{q}) 2(2 \pi \varepsilon)^{1 / 2}\left(\mathrm{R}_{\mathrm{G}} \mathrm{GL}_{\mathrm{n}}\right)^{3 / 2} \int_{\mathrm{d} k_{\theta} \mathrm{k}_{\theta}} 2 \mathrm{I}\left(\mathrm{k}_{\theta}\right) \mathrm{F}\left(\Omega\left(\mathrm{k}_{\theta}\right)\right) \int \mathrm{dx} \exp \left(-\mathrm{x}^{2} / \mathrm{w}_{\mathrm{n}}{ }^{2}\right) \text {. }
\end{aligned}
$$

Using Eq. (25) and after the radial integration over the mode width, the $\mathrm{k}_{\theta}$-integration becomes

$$
\begin{gathered}
\pi^{1 / 2}\left(\beta^{L} / S \beta^{I C S}\right)\left(C_{s} \rho_{s} / L_{n}\right)^{4} k_{M}-1 \int_{d \theta \theta}-2\left(1+\theta^{2}\right)^{-15 / 2} \mid 1-\theta^{2} \beta \\
\exp \left(-R / G L_{n}\left(1+\theta^{2}\right)\right)\left(1-\left(1+\theta^{2}\right)^{-1 / 2}+\eta_{e}\left(R\left(1+\theta^{2}\right)^{-1 / 2} / \mathrm{GL}_{n}-3 / 2\right)\right\} \\
\int_{\theta} d \phi \phi^{-1}\left(1+\phi^{2}\right)^{-1 / 2} \exp \left(-R / G_{n}\left(1+\phi^{2}\right)\right)\left(1-\left(1+\phi^{2}\right)^{-1 / 2}+\eta_{e}\left(R\left(1+\phi^{2}\right)^{-1 / 2} / \mathrm{GL}_{n}-3 / 2\right)\right\},
\end{gathered}
$$

where $\theta=k_{\theta} 1 / k_{M}, \phi=k_{\theta} / k_{M}$, and we have changed the order of integration. Since $\phi<\theta$ $<1$ and the integrand of $\theta$-integration vanishes rapidly as $\theta \Rightarrow 0$, for realistic finite values of $\eta_{e}$, the $\phi$-integration can be approximated by ignoring $\phi^{2}$ in comparison to 1 without significantly influencing the final result. Then, the last factor of Eq. (36) becomes, after an elementary integration, $\exp \left(-R / G L_{n}\right) \eta_{e}\left(R / G L_{n}-3 / 2\right)\left(-\ln \left(k_{L} / k_{M}\right)+\ln \theta\right)$. Now, it is straightforward to perform the $\theta$-integration which is dominated by the small- $\theta$ behavior. Finally the expression for the particle flux is

$$
\begin{aligned}
& \Gamma_{\mathrm{e}} \equiv-D^{\operatorname{eff}} \mathrm{dn} \mathrm{n}_{\mathrm{d}} / \mathrm{dx} \\
& =-\left(2^{7} \varepsilon / 15\right)\left(\mathrm{q}^{2} / \mathrm{S}^{2}\right)\left(\mathrm{T}_{\mathrm{e}} / \mathrm{T}_{\mathrm{i}}\right) \eta_{\mathrm{e}}\left(1+5 \eta_{\mathrm{i}} / 4\right)^{-1}\left[\mathrm{k}_{\mathrm{M}} / \mathrm{k}_{\mathrm{L}}-\ln \left(\mathrm{k}_{\mathrm{M}} / \mathrm{k}_{\mathrm{L}}\right)-1\right]\left(\mathrm{k}_{\mathrm{M}} \mathrm{P}_{\mathrm{S}}\right) \\
& \left(R / L_{n}\right)^{2}\left(R / G L_{n}\right)^{3}\left(R / G L_{n}-3 / 2\right)^{2} \exp \left(-2 R / G L_{n}\right) \rho_{s} L_{T e}{ }^{-1}\left(c T_{e} / e B\right) d_{0} / d x \text {. }
\end{aligned}
$$

The electron thermal flux is calculated by the same method. Since the contribution from the nonlinear trapped electron scattering is negligible due to a small fluctuation level at high $\mathrm{k}_{\theta}$ $\left(k_{\theta}>k_{M}\right)$, the trapped electron thermal flux is given by

$$
\mathrm{Q}_{e}=\operatorname{Re} \Sigma_{k} \mathrm{ik}_{\theta}\left(\mathrm{cT}_{\mathrm{e}} / \mathrm{eB}_{0}\right) \Phi_{-k} \iint_{\mathrm{d}} 3_{\mathrm{v}} \mathrm{E} \mathrm{h}_{\mathbf{k}}(1) \text {. }
$$

Since the resonant trapped electrons are transported preferentially, the procedure of calculating 
$Q_{e}$ is very similar to the one used for the particle flux calculation. The only difference is that the energy moment rather than the density moment is involved in the integration here. The resonant energy of the trapped electron is given by $E_{\text {Tes }}=\left(R / G_{n}\left(1+k_{\theta}{ }^{2} / k_{M}{ }^{2}\right)\right) T_{e}$. Thus, there exists the following relation between $Q_{e}$ and $\Gamma_{e}$,

$$
\mathrm{Q}_{\mathrm{e}}=\left(\mathrm{R} / \mathrm{GL}_{\mathrm{n}}\right) \mathrm{T}_{\mathrm{e}} \Gamma_{\mathrm{e}}
$$

The resulting trapped el:ctron thermal flux can be explicitly written as

$$
\begin{aligned}
& \mathrm{Q}_{e} \equiv-\chi_{e} e^{e f f_{0} T_{e} / d x} \\
& =-\left(2^{7} \varepsilon / 15\right)\left(q^{2} / S^{2}\right)\left(T_{e} / T_{i}\right)\left(1+5 \eta_{i} / 4\right)^{-1}\left[k_{M} / k_{L}-\ln \left(k_{M} / k_{L}\right)-1\right]\left(k_{M} P_{S}\right) \\
& \left(R / L_{n}\right)^{2}\left(R / G L_{n}\right)^{4}\left(R / G L_{n}-3 / 2\right)^{2} \exp \left(-2 R / G L_{n}\right) \rho_{S} L_{T} e^{-1}\left(c T_{e} / e B\right) n_{0} d T_{e} / d x .
\end{aligned}
$$

The ion thermal flux is also calculated in this Section. As mentioned before, for most of the $k_{\theta}$-range of interest where the fluctuation is appreciable, the magnetic shear-induced ion Landau damping does not affect the nature of fluctuation structure. Hence, the linear ion Landau damping has no significant direct effect on the ion thermal transport, and the ion Compton scattering plays a dominant role in determining the amount of ion heat flux driven by the fluctuations. Keeping only the dominant nonlinear (ion Compton scattering) contribution gives,

$$
Q_{i}=-\Sigma_{k} k_{\theta}\left(\mathrm{cT}_{e} / e B_{0}\right) \Phi_{-k} \operatorname{Im} \int d^{3} v E J_{0} g_{k}(3),
$$

where the expression ior $\mathrm{g}^{(3)}$ is available from Eq. (13). For relatively long wavelength fluctuations, the Bessel function, $\mathrm{J}_{0}$, can be approximated by 1 , and the expression for the velocity space resonant integral for the (axwellian back-ground distribution function becomes

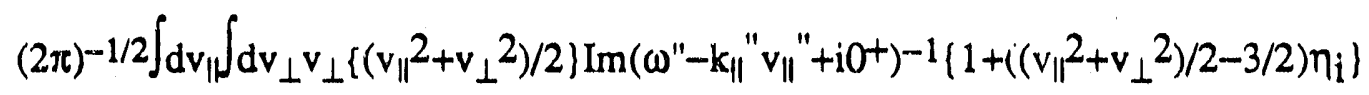

$\exp \left\{-\left(v_{\|}{ }^{2}+v_{\perp}{ }^{2}\right) / 2\right\}$, where $v_{\|}$and $v_{\perp}$ have been normalized to $v_{\mathrm{Ti}}$. Using Premelji's formula for the wave-particle resonance, it is straight-forward to evaluate the integral which yields $(\pi / 2)^{1 / 2}\left(\left|k_{\|}\right| " v_{T i}\right)^{-1}\left(\eta_{i}\left(|\omega "| 2 / 2\left(\left|k_{\|}\right| " v_{T i}\right)^{2}\right)^{2}+\left(1+|\omega "| 2 / 2\left(\left|k_{\|}\right| " v_{T_{i}}\right)^{2}\right)\left(1+\eta_{i} / 2\right)\right\}$ $\left.\exp (-\mid \omega)^{\prime \prime} \mid 2 / 2\left(k_{\|} \mid{ }^{\prime \prime} v_{T i}\right)^{2}\right\}$. The remaining $d x$ and $d k_{\theta}$ integrals for mode summations are similar 
to the previous calculations for $\Gamma_{e}$ and $Q_{e}$, and are not repeated here. The final expression for $Q_{i}$ is given by

$$
\begin{aligned}
& \mathrm{Q}_{\mathrm{i}} \equiv-\chi_{\mathrm{i}} \text { eff } \mathrm{n}_{0 \mathrm{~d} \mathrm{~T}_{\mathrm{i}} / \mathrm{dx}} \\
& =2.75\left(1+. .93 \eta_{\mathrm{i}}\right)\left(1+1.25 \eta_{\mathrm{i}}\right)^{-1} \mathrm{~T}_{\mathrm{i}} \Gamma_{\mathrm{e}} .
\end{aligned}
$$

Finally, the anomalous heat exchange rate between two species in the presence of electrostatic fluctuations can be calculated using

$$
\partial / \partial t W_{j}=e_{j} \operatorname{Re} \Sigma_{k} \Phi_{-k} \partial / \partial t \int_{d} 3 v\left(h_{k}(1)+h_{k}{ }^{(3)}\right)_{j}
$$

with $\Sigma_{j} \partial / \partial t W_{j}=0$. For electrons, the anomalous "cooling" rate is then roughly estimated to be given by $Q_{e i} \equiv-T_{e} \Gamma_{e} / L_{n}$. This expression, which is similar to the result for dissipative trapped electron modes, 38 shows that the electrons lose energy to the fluctuation (the wave) while the ions gain energy from the fluctuation at the same rate thereby keeping the fluctuation amplitude at the saturated level.

\section{Discussions}

In this paper, we have developed a weak turbulence theory of CTEM in toroidal geometry in the presence of both ion and electron temperature gradients treating ion Compton scattering and the trapped electron scattering on an equal footing. It is found that both nonlinearities are important in determining the spectral shape of the saturated potential fluctuations. This shape is given by an approximate power law, $k_{\theta}{ }^{-3}$, and extends from $k_{\theta} \rho_{S}$ $\equiv\left(L_{n} / R\right)^{1 / 2}$ to $k_{\theta} \rho_{s} \cong 1$. The corresponding fluctuation amplitude is found to be smaller than the mixing length estimate. Fluctuation-induced anomalous particle and heat fluxes have also been obtained and are again found to be smaller than the mixing length estimates. As indicated by Eqs. (37), (40) and (42), various fluxes obey similar scalings. The plasrna confinement is 
predicted to improve with i) higher $T_{i} / T_{e}$, ii) more peaked density profile, iii) larger aspect ratio, and iv) higher plasma current. In addition, the results indicate a significant dependence of transport on the electron temperature gradient. This might be relevant to the rigidity of the electron temperature profiles 18 observed in many tokamak plasmas. 19

The favorable dependence on $T_{i} / T_{e}$ is a consequence of ion Compton scattering (which is obviously stronger for higher $v_{\mathrm{Ti}}$ ) being an important nonlinear saturation mechanism. This favorable dependence on $\mathrm{T}_{\mathrm{i}} / \mathrm{T}_{\mathrm{e}}$ is almost universally obtained in hot-ion mode operation of the large tokamaks $6,39,40$ where $T_{i} / T_{e}$ can be as large as $3-4.6,39$ In contrast, many previous theories of drift wave turbulence were developed using the familiar cold ion $\left(\mathrm{T}_{\mathrm{j}} / \mathrm{T}_{\mathrm{e}} \ll 1\right)$ assumption.

The calculated confinement improvement with density peaking 41 is a consequence of the particular destabilizing mechanism 8 considered in this paper, i.e., the precession drift resonance of the trapped electrons with waves. As the density profile gets more peaked, the wave frequency $\left(\approx \omega_{* \mathrm{e}}\right)$ increases and detunes from the precession drift frequency of thermal trapped electrons. From the experimental side, the confinement improvement with density peaking has been a familiar trend observed in a number of pellet experiments $42-44$ and has frequently been quoted as evidence supporting the relevance of ITG-modes. However, it should be remembered that the present analysis is valid in a low collisionality (CTEM) regime. In this sense, it is more meaningful to compare the present results to TFTR auxiliarily heated plasmas where the confinement improvement from L-mode to Supershot can be characterized by the density peakedness. 6,45 It is also interesting to note that for these shots, the dependence of ITG-mode threshold conditions on the density gradient scale length $\left(L_{n}\right)$ is rather weak due to high values of $T_{i} / T_{e} \cdot 46,47$

The aspect ratio $\left(\varepsilon^{-1}\right)$ dependence found in the present calculations comes from the fact that the fraction of trapped electrons is proportional to $\varepsilon^{1 / 2}$. Our weak turbulence analysis 
indicates that the anomalous particle and heat fluxes are proportional to $\varepsilon$, while simple mixing length estimates ${ }^{3-5}$ predict an $\varepsilon^{1 / 2}$ dependence. Recent results from TFTR 48 tend to be in somewhat better agreement with our result.

The absence of a significant scaling of local transport with current $\left(\mathrm{I}_{\mathrm{p}}\right)$ and/or the edge safety factor $(q(a))$ in most drift-type microinstability theories has been a persistent and challenging problem. This is a very relevant issue because a strong scaling of this kind has in fact been found in empirically-deduced global energy confinement times 49,50 for auxiliary-heated tokamak plasmas. It is, therefore, important to note that the results of our present analysis of collisionless trapped-electron modes does indeed exhibit a significant local $\mathrm{q}(\mathrm{r})$ dependence. As pointed out in Ref. 21, such behavior can be explained in part by the fact that as $q(a)$ (and the associated local value of $S$ ) increases, the equilibrium value for the trapped-electron orbit-averaged magnetic precession drifts $(\propto \mathrm{G})$ becomes increasingly unfavorable for $\mathrm{q}(\mathrm{a})$ values up to 7 or so. Moreover, the favorable transit ion nonlinear (as well as linear) Landau damping effects are likewise enhanced for lower $q(r)$ values. While the present results are suggestive of the proper trend, care should be exercised in applying them to specific experimental scenarios. For example, although the confinement zone of TFTR supershots falls well within the CTEM regime, no appreciable current dependence is observed. This could be due to the fact that the density profiles in these discharges were observed to steepen more as $q(a)$ increases (i.c., $I_{p}$ decreases). Hence, the unfavorable influence of decreasing $I_{p}$ might be offset by the aforementioned predicted improvement of confinement with density peaking.

In the present study a significant dependence of transport on the electron temperature gradient $\left(\nabla T_{e}\right.$ ) is found (i.e. ; $Q_{e}, \Gamma_{e}$, and $Q_{i} \propto L_{T e}{ }^{-2}$ ). Such a trend is naturally expected since the electron temperature gradient can often be a more effective source of free energy than the density gradient in driving microinstabilities. It is well known 51 that if the electron 
temperature gradient is ignored for these modes, A much weaker version of these instabilities (requiring finite lon gyroradius reduction of the drift frequency) results. Accorcingly, it is important to exercise caution in the application of those drift wave turbulence theories which ignore the electron temperature gradient to the interpretation of confinement trends observed in tokamak plasmas.

\section{Acknowledgments}

The authors would like to thank L. Chen, P. H. Diamond, F. W. Perkins, M. H. Redi, G. Rewoldt, H. Shirai, and M. Zamstorff for useful conversations.

This work was supported by United States Department of Energy Contract No. DE-AC02-76-CHO-3073. 


\section{References}

1. W. M. Tang, Nucl. Fusion 18, 1089 (1978).

2. D. W. Ross, P. H. Diamond, J. F. Drake, F. L. Hinton, F. W. Perkins, W. M. Tang, R. E. Waltz, S. J. Zweben, Univ. of Texas Fusion Research Center Report, FRCR \#295 (1987).

3. F. W. Perkins, in Heating in Toroidal Plasmas (Proc. 4th Int. Symp. Rome, 1984), Vol.2, Int. School of Plasma Physics, Varenna (1984) 977.

4. M. H. Redi, W. M. Tang, P. C. Efthimion, D. R. Mikkelsen, and G. L. Schmidt, Nucl. Fusion 2 7, 2001(1987).

5. R.R. Dominguez and R. E. Waltz, Nucl. Fusion 27, 65 (1987).

6. M.C. Zarnstorff $e_{\imath}$ al., in Proceedings of 16 th European Conference on Controlled Fusion and Plasma Heating, Vol. 1, pp.35 (1989).

7. K. H. Burrell et al., in Proceedings of 17 th European Conference on Controlled Fusion and Plasma Heating, Vol. 1, pp.271 (1990).

8. J. C. Adam, W. M. Tang, and P. H. Rutherford, Phys. Fluids 19, 561 (1976).

9. G. Rewoldt, W. M. Tang, and E. A. Frieman, Phys. Fluids 20, 402 (1977).

10. C. Z. Cheng and L. Chen, Nucl. Fusion 21, 403 (1981).

11. G. Rewoldt and W. M. Tang, Phys. Fluids B 2, 318 (1990).

12. C. Z.Cheng and K. T. Tsang, Nucl. Fusion 21, 643 (1981).

13. L. Chen, R. L. Berger, J. G. Lominadze, M. N. Rosenbluth, and P. H. Rutherford, Phys. Rev. Lett 39, 754 (1977).

14. A. Rogister and G. Hasselberg, Phys. Fluids 26, 1467 (1983).

15. F. Y. Gang, P. H. Diamond, and M. N. Rosenbluth, Submitted to Phys. Fluids B (1990).

16. L. D. Pearlstein and H. L. Berk, Phys, Rov. Lett 23, 220 (1969).

17. P. L.Similon and P. H. Diamond, Phys. Fluids 27, 916 (1984). 
18. B. Coppi, Comm. Plasma Phys. Controll. Fusion 5, 261 (1980).

19. R. J. Goldston and TFTR Group, in Plasma Phys. Controlled Fusion Research (International Atomic Energy Agency, Vienna, 1987), Vol. I, p.75.

20. B. Coppi and G.Rewoldt, Advances in Plasma Physics 6, 421 (1976).

21. W. M. Tang, G.Rewoldt, and T. S. Hahm, Proceedings of Sherwood Theory Conference, Williamsburg, VA (1990) Paper 1B1.

22. L. Chen and C. Z. Cheng, Phys. Fluids 23, 2242 (1980).

23. G.Rewoldt, W. M. Tang, and E. A. Frieman, Phys. Fluids 20, 402 (1977).

24. B. Coppi and G. Rewoldt, Phys. Lett 49 A, 36 (1974).

25. B. Coppi and G. Rewoldt, Phys. Rev. Lett 33, 1329 (1974).

26. B. Coppi and F. Pegoraro, Nucl. Fusion 17, 963 (1977).

27. R. Z. Sagdeev, and A. A. Galeev, in Nonlinear Plasma Theory, edited by T. M. O'Neil and D. L. Book ( Benjamin, NewYork, 1969).

28. E. A. Frieman and L. Chen, Phys. Fluids 25, 502 (1982).

29. W. Horton, Jr. et al., in Plasma Phys. Controlled Fusion Research (International Atomic Energy A gency, Vienna, 1975), Vo!. I, p. 541.

30. W. M. Tang et al., in Plasma Phys. Controlled Fusion Research (International A.tomic Energy Agency, Vienna, 1977), Vol. II, p.489.

31. J. A. Krommes, Phys. Fluids 23, 736 (1980).

32. T. S. Hahm and W. M. Tang, Phys. Fluids B 2,1815 (1990).

33. P. H. Diamond and M. N. Rosenbluth, Phys. Fluids 19, 561 (1976).

34. C. Surko and R. Slusher, Science 221, 817 (1983).

35. T. Crowley and E. Mazzucato, Nucl. Fusion 25, 507 (1985).

36. N. L. Bretz, R. Nazikian, and K. L. Wong, in Proceedings of 17th European Conference on Controlled Fusion and Plasma Heating, Vol. IV, pp.1544 (1990). 
37. G. Dodel, E. Holzhauer, L. Giannone, H. Niedermeyer, J. Gernhardt, and Asdex Team, in Proceedings of 17th European Conference on Controlled Fusion and Plasma Heating, Vol. I, pp.206 (1990).

38. Wallace M. Manheimer, E. Ott, and W. M. Tang, Phys. Fluids 20, 806 (1977).

39. A. Tanga et al., in Proceedings of 17 th European Conference on Controlled Fusion and Plasma Heating, Vol. I, pp.259 (1990).

40. JT-60 Team, Review of JT-60 Experimental Results from Jan. to Oct. 1989, Rep. JAERI-M 90-066, Ibaraki-ken, Japan (1990) pn، 146-177.

41. T.S. H.ahm and W.M. Tang, in "Structures in Confineci Plasmas" Proceedings of Workshop of US-Japan Joint Institute for Fusion Theory Program, pr. 55 (1990).

42. S. M. Wolf, and M. Greenwald, Nucl. Fusion 26, 329 (1986).

43. D. L. Brower, W. A. Peebles, S. K. Kim, N. C. Luhman, Jr., W. M. Tang, and P. E. Phillips, Phys. Rev. Lett 59, 49 (1987).

44. R. Hawryluck and TFTR Group, in Plasma Physics and Controlled Fusion Research (International Atomic Energy Agency, Vicnna, 1987), Vol. I, p.51.

45. H. K. Park, R. J. Goldston, and G. Taylor, in Proceedings of 4th International Symposium on Laser-Aided Plasma Diagnostics, Fukuoka, Japan (LAPD IV-89/II-3) pp.17 (1989).

46. T. S. Hahm and W. M. Tang; Phys. Fluids B 1, 1185 (1989).

47. F. Romanelli, Phys. Fluids B 1, 1018 (1989).

48. L.R. Grisham et al., in Proceedings of 17 th European Conference on Controlled Fusion and Plasma Heating, Vol. I, pp.146 (1990).

49. R. J. Goldston, Plasma Phys. Controlled Fusion 26, 87 (1984).

50. S. Kaye, Phys. Fluids 28, 2327 (1985).

51. C.S. Liu, M.N. Rosenbluth, and W.M. Tang, Phys. Fluids 19, 1040 (1976). 


\section{Figures}

FIG. 1. Plot of spectral intensity, $\mathrm{I}\left(\mathrm{k}_{\theta}\right)$, in logarithmic scale. Three different asymptotic regions in $k_{\theta}$ are identified. The region " $a$ " corresponds to an extremely long wavelength regime where fluctuations are suppressed due to the trapped electron scattering.

The region " $b$ " corresponds to a long wavelength regime where most of the fluctuation energy is populated and $I\left(k_{\theta}\right)$ decays according to a power law, $k_{\theta}{ }^{-3}$, given in Eq. (27). Finally, region "c" corresponds to a relatively shorter wavelength regime, $\mathrm{k}_{\theta}>\mathrm{k}_{\mathrm{M}}$, where $\mathrm{I}\left(\mathrm{k}_{\theta}\right)$ decays more steeply. 


\section{EXTERNAL DISTRIBUTION IN ADDITION TO UC-420}

Dr. F. Paoloni, Univ. of Wollongong, AUSTRALIA

Prof. M.H. Brennan, Univ, of Sydney, AUSTRALIA

Plasma Fesearch Lab., Australian Nat. Univ., AUSTRALJA

Prot. I.R. Jones, Flinders Univ, AUSTRALIA

Prof. F. Cap, Inst. for Theoretical Physics, AUSTRIA

Prof. M. Heindler, Instui fur Theoretische Physik, AUSTRIA

Prof. M. Goossens, Astronomisch insticuUt, BELGIUM

Ecola Royale Militaire, Lab. de Phy. Plasmas, BELGIUM

Commission-European, DG. XII-Fusion Prog., BEL.GIUM

Prot. R. Bouciquie, Rilksuniversiteit Gent, BELGIUM

Dr. P.H. Sakanaka, Instituto Fisica, BRAZIL

Instituto De Pesquisas Espaciais-INPE, BRAZIL

Documents Office, Atomic Energy of Canada Lid., CANADA

Dr. M.P. Eachynski, MPB Tochnologies, Inc., CANADA

Dr. H.M. Skarsgard, Univ. of Saskatchewan, CANADA

Prot. J. Teichmann, Univ. of Montreal, CANADA

Prot. S.R. Sreonivasan, Univ. of Calgary, CANADA

Prof. T.W. Johnston, INRS-Energie, CANADA

Dr. R. Bolton, Centre canadien de husion magnétique, CANADA

Dr. C.R. James., Univ, of Alberta, CANADA

Dr. P. Lukac, Komenskeho Universzita, CZECHOSLOVAKIA

The Librarian, Culham Laboratory, ENGLAND

Library, R6Y, Ruthertord Appleton Laboratory, ENGLAND

Mrs, S.A. Hutchinson, JET Library, ENGLAND

P. Măhönen, Univ. of Heisinkj, FINLAND

C. Mouttet, Lab. de Physique des Milieux lonisés, FPANCE

J. Radet, CEN/CADARACHE - Bat 506, FRANCE

Ms. C. Rinni, Univ, of loannina, GREECE

Or. T. Mual, Acadomy Bibliographic Ser, HONG KONG Preprint Library, Hungarian Academy of Sci., HUNGARY

Dr. B. Das Gupta, Saha Inst. of Nudear Physica, INDIA

Dr. P. Kaw, Inst. for Plasma Research, INDIA

Dr. P. Rosenau, Israel Inst. of Technology, ISRAEL. Librarian, Intemational Center for Theo Physics, ITALY Miss C. De Palo, Associazione EURATOM-ENEA, ITALY Or. G. Grosso, Istituto di Fisica del Plasma, ITALY Dr. H. Yamato, Toshiba Res \& Deved Center, JAPAN

Prof. I. Kawakami, Abomic Energy Res.Inst., JAPAN

Prof. K. Nishikawa, Hirashima Univ., JAPAN
Director, Japan Atomic Energy Researcti inst., JAPAN

Prof. S. Itoh, Kyushu Univ., JAPAN

Data and Planning Center, Nagoya Univ., JAPAN

Prof. S. Tanaka, Kyoto Univ., JAPAN

Library, Kyoto Univ., JAPAN

Prot. N. Inoue, Univ. of Tokyo, JAPAN

S. Mori, Tochnical Advisor, JAERI, JAPAN

O. Mitarai, Kumamoto Inst. of Technology, JAPAN

H. Jeong, Korea Advanced Energy Research Inst., KOREA

Prot. D.I. Choi, The Korea Adv. Inst. of Sd. Tech., KOREA

Prof. B.S. Liley, Univ. of Waikato, NEW ZEALAND

Inst. of Plasma Physics, PEOPLE'S REPUBLIC OF CHINA

Librarian, Inst, of Physics, PEOPLE'S REPUBLIC OF CHINA

Library, Tsinghua Univ., PEOPLE'S REPUBLIC OF CHINA

Z. L, S.W. Inst Physics, PEOPLE'S REPUBLIC OF CHINA

Prot. J.A.C. Cabral, Instituto Superior Tecnico, PORTUGAL

Dr. O. Petrus, AL I CUZA Univ., ROMANIA

Dr. J. de Villiers, Fusion Studies, AEC, S. AFRICA

Prof. M.A. Hellberg, Univ, of Natal, S. AFRiCA

C.I.E.M.A.T, Fusion Division Library, SPAIN

Dr. L Stonflo, Univ. of UMEA, SWEDEN

Library, Royal Inst. of Technology, SWEDEN

Prof. H. Wilhelmsen, Chaimers Univ, of Tech., SWEDEN

Centro Phys. Des Plasmas, Ecolo Polytech, SWITZERLAND Bibliothook, Inst. Voor Plasma-Fysica, THE NETHERLANDS M. Durguth Vico Chaiman, Middlo East Toch. Univ., TURKEY Dr. D.D. Ryutov, Siberian Branch of Academy of Sci., USSR

Dr. G.A. Eliseev, Kurchatov Inst. USSR

Librarian, The Ukr.SSR Academy of Sciences, USSR

Dr. L.M. Kovrizhnykh, Inst of Genoral Physics, USSR Kemfor schungsanlage GmbH, Zentralbibliothek, W. GEAMANY Bibliothek, Inst. Fü Plasmaforschung, W. GERMANY Prof. K. Schindler, Ruhr-Univorsitát BOchum, W. GERMANY Dr. F. Wagner, (ASDEX), Max-Planck-Institut, W. GERMANY Librarian, Max-Planck-Institut, W. GERMANY

Prof. R.K. Janev, Inst. of Physics, YUGOSLAVIA 

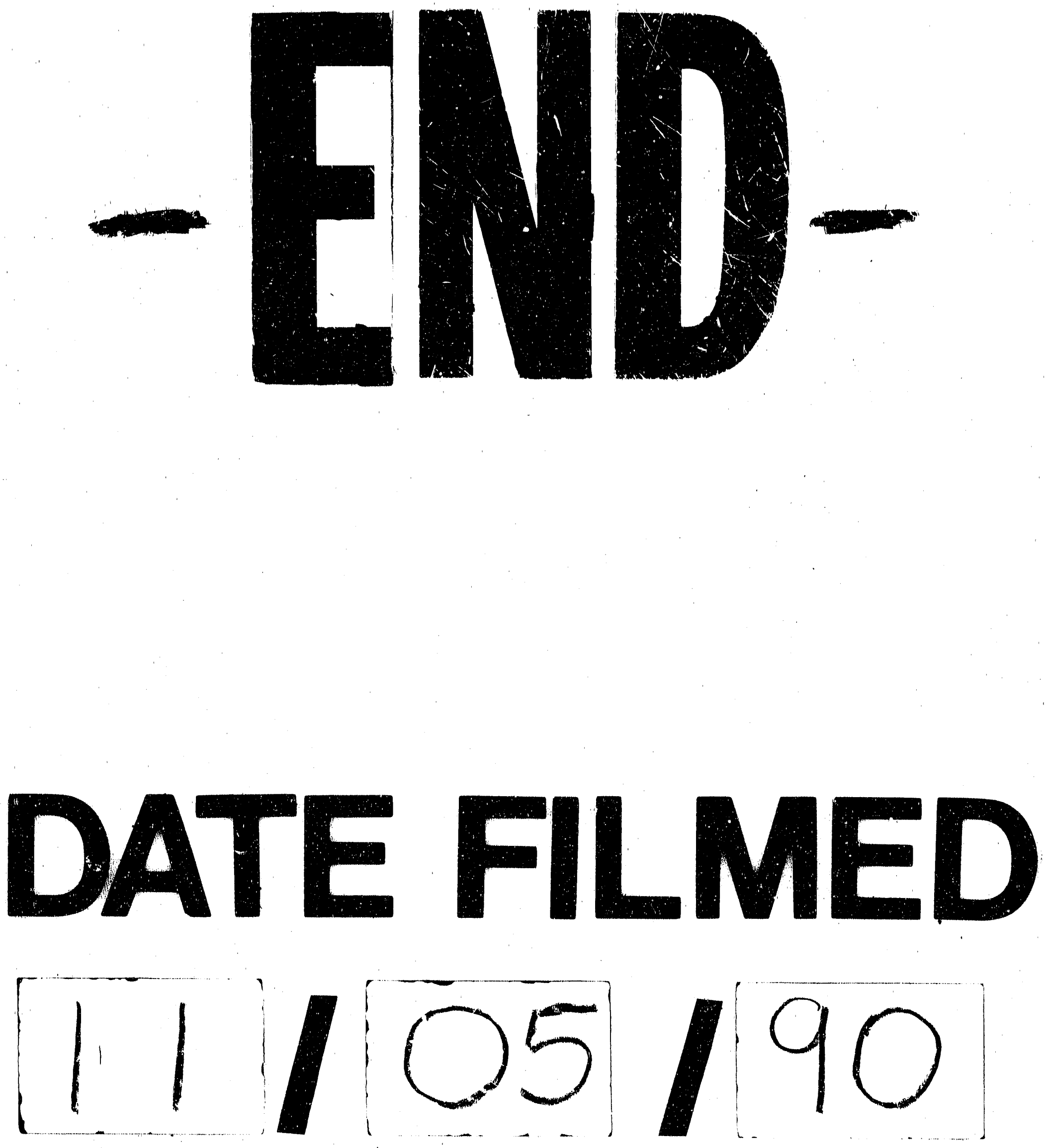
